\title{
Herschel observations of gamma-ray burst host galaxies: implications for the topology of the dusty interstellar medium ${ }^{\star}$
}

\author{
P. Schady ${ }^{1}$, S. Savaglio ${ }^{1,2,3}$, T. Müller ${ }^{1}$, T. Krühler ${ }^{4}$, T. Dwelly ${ }^{1}$, E. Palazzi ${ }^{5}$, L. K. Hunt ${ }^{6}$, J. Greiner ${ }^{1}$, H. Linz $^{7}$, \\ M. J. Michałowski ${ }^{8,9}$, D. Pierini ${ }^{10,11,12, \star \star}$, S. Piranomonte ${ }^{12}$, S. D. Vergani ${ }^{13,14}$, and W. K. Gear ${ }^{15}$ \\ 1 Max-Planck-Institut für Extraterrestrische Physik, Giessenbachstraße, 85748 Garching, Germany \\ e-mail: pschady@mpe.mpg.de \\ 2 Physics Department, University of Calabria, 87036 Arcavacata di Rende, Italy \\ 3 European Southern Observatory, 85748 Garching, Germany \\ 4 European Southern Observatory, Alonso de Córdova 3107, Vitacura, Casilla 19001 Santiago 19, Chile \\ 5 INAF - IASF Bologna, via Gobetti 101, 40129 Bologna, Italy \\ 6 INAF - Osservatorio Astrofisico di Arcetri, Largo E. Fermi, 5, 50125 Firenze, Italy \\ 7 Max-Planck-Institut für Astronomie, Königstuhl 17, 69117 Heidelberg, Germany \\ 8 Sterrenkundig Observatorium, Universiteit Gent, Krijgslaan 218-S9, 9000 Gent, Belgium \\ 9 SUPA (Scottish Universities Physics Alliance), Institute for Astronomy, University of Edinburgh, Royal Observatory, Edinburgh, \\ EH9 3HJ, UK \\ 10 Institut de Recherche en Astrophysique et Planétologie, CNRS-Université Paul Sabatier Toulouse, 9 avenue du Colonel Roche, \\ 31028 Toulouse, France \\ 11 Centre de Physique des Particules de Marseille, Aix Marseille Université, 168 avenue de Luminy, 13009 Marseille, France \\ 12 INAF - Osservatorio Astronomico di Roma, via di Frascati, 33, 00040 Monteporzio Catone, Italy \\ 13 GEPI - Observatoire de Paris, CNRS UMR 8111, Univ. Paris-Diderot, 5 place Jules Jannsen, 92190 Meudon, France \\ 14 INAF/Osservatorio Astronomico di Brera, via Emilio Bianchi 46, 23807 Merate (LC), Italy \\ 15 School of Physics and Astronomy, Cardiff University, 5 The Parade, Cardiff, Wales CF24 3YB, UK
}

Received 29 April 2014 / Accepted 18 August 2014

\section{ABSTRACT}

\begin{abstract}
Long-duration gamma-ray bursts (GRBs) are indisputably related to star formation, and their vast luminosity in gamma rays pinpoints regions of star formation independent of galaxy mass, out to the epoch of re-ionisation. As such, GRBs provide a unique tool for studying star forming galaxies out to high- $z$ independent of luminosity. Most of our understanding of the properties of GRB hosts (GRBHs) comes from optical and near-infrared follow-up observations, and we therefore have relatively little knowledge of the fraction of dust-enshrouded star formation that resides within GRBHs. Currently $20 \%$ of GRBs show evidence of significant amounts of dust within their host galaxy along the GRB line of sight, and these GRBs tend to reside within redder and more massive galaxies than GRBs with optically bright afterglows. In this paper we present Herschel observations of five GRBHs with evidence of being dust-rich, targeted to understand the properties of the dust attenuation within GRBs better. Despite the sensitivity of our Herschel observations, only one galaxy in our sample was detected (GRBH 070306), for which we measure a total star formation rate of $\sim 100 M_{\odot} \mathrm{yr}^{-1}$, and which had a relatively high stellar mass $\left(\log \left[M_{*}\right]=10.34_{-0.04}^{+0.09}\right)$. Nevertheless, when considering a larger sample of GRBHs observed with Herschel, it is clear that stellar mass is not the only factor contributing to a Herschel detection, and significant dust extinction along the GRB sightline $\left(A_{V, \mathrm{GRB}}>1.5 \mathrm{mag}\right)$ appears to be a considerably better tracer of GRBHs with a high dust mass. This suggests that the extinguishing dust along the GRB line of sight lies predominantly within the host galaxy ISM, and thus those GRBs with $A_{V, \mathrm{GRB}}>1 \mathrm{mag}$ but with no host galaxy Herschel detections are likely to have been predominantly extinguished by dust within an intervening dense cloud.
\end{abstract}

Key words. galaxies: high-redshift - gamma-ray burst: general - dust, extinction - infrared: galaxies - submillimeter: galaxies galaxies: star formation

\section{Introduction}

Ever since the connection between long-duration gamma-ray bursts (GRBs) and massive star formation was firmly established (Galama et al. 1999; Hjorth et al. 2003), much attention has been focused on using GRBs to trace the cosmic star formation history (SFH). However, such ambitions have been hindered by indications that GRBs may not be indiscriminate tracers of star formation (Kistler et al. 2009; Butler et al. 2010; Wanderman \& Piran 2010; Robertson \& Ellis 2012; but see Michałowski et al. 2012b)

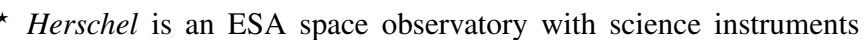
provided by European-led Principal Investigator consortia and with important participation from NASA.

$\star \star$ Visiting astronomer.
}

and that further factors may play a dominant role in regulating the GRB formation rate (e.g. metallicity, the initial mass function; IMF). The GRB "collapsar" model predicts a metallicity threshold of $\sim 0.3 Z_{\odot}$, above which massive stars cannot collapse to form a GRB (MacFadyen \& Woosley 1999; Langer \& Norman 2006), and there are observations that similarly point to GRBs occurring predominantly within low-metallicity environments (Fruchter et al. 2006; Wolf \& Podsiadlowski 2007; Modjaz et al. 2008; Levesque et al. 2010b; Graham \& Fruchter 2013). There are, however, examples of GRB hosts (GRBHs) with near-solar metallicity or above (Graham et al. 2009; Levesque et al. 2010b; Savaglio et al. 2012; Elliott et al. 2013), and rather than metallicity, other environmental properties have been said to play a more critical role in the formation of GRBs, such 
as the SFR per unit stellar mass (i.e. specific SFR or sSFR) (e.g. Mannucci et al. 2011; Kocevski \& West 2011; Kelly et al. 2014; Hunt et al. 2014) or a high interstellar medium density (Michałowski et al. 2014).

Most of our understanding of the environmental properties of GRBHs comes from photometric and spectroscopic observations of bright, dust-poor galaxies, which make up the bulk of the population at $z<1$ (Savaglio et al. 2009; Graham $\&$ Fruchter 2013), and for which data are more readily available. Over the last decade, great emphasis has been put on extending the GRBH detection rate out to higher redshifts, and to more dust-rich systems. The rapid, sub-arcsecond positions provided by the GRB-dedicated NASA Swift mission (Gehrels et al. 2004), along with the commissioning of numerous nearinfrared (NIR) facilities for GRB follow-up observations, such as PAIRITEL (Bloom et al. 2006), GROND (Greiner et al. 2008) and more recently RATIR (Butler et al. 2012), have resulted in an increase in the fraction of detected afterglows that are significantly dust-extinguished (i.e. rest frame $V$-band dust extinction $A_{V, \mathrm{GRB}}>1.0 \mathrm{mag}$ ) (e.g. Greiner et al. 2011). These reddened GRBs tend to reside within galaxies that are more massive and luminous than the more frequently observed hosts of GRBs with optically bright afterglows (Krühler et al. 2011; Hunt et al. 2011; Svensson et al. 2012; Perley et al. 2013).

Although it is still debated whether the fraction of more massive, dustier hosts are as numerous as would be expected if GRBs directly follow the star formation activity (Perley et al. 2013; Hunt et al. 2014), their detection certainly does present challenges to the GRB “collapsar" model, which requires a progenitor star metallicity cut-off. Studying the abundance and properties of dust within GRB host galaxies is thus important not only in the use of GRBs as cosmological tools, but also for our understanding of the progenitors and environmental factors that produce these catastrophic explosions. There have now been several published works that looked into the properties of the more massive and dust-rich GRB host galaxies (e.g. Krühler et al. 2011; Hunt et al. 2011; Perley et al. 2013; Rossi et al. 2012). However, for the most part these investigations have not included observations of the host galaxy dust emission, which probe the obscured star formation and can contain a significant fraction of the galaxy energy density.

We have used the infrared Herschel satellite (Pilbratt et al. 2010) to study the dust emission properties of a small sample of five GRBHs that we considered good candidates of containing appreciable amounts of dust. With a sample of five host galaxies, our aim is to reach a greater understanding of the dust properties within this dustier and important class of GRBHs, rather than to draw any quantifiable conclusions on the relation between GRBs and the cosmic SFR. Our Herschel PACS and SPIRE observations span the wavelength range from $100 \mu \mathrm{m}$ to $500 \mu \mathrm{m}$, which for our sample (at $0.8<z<1.5$ ) and for typical dust temperatures of $35 \mathrm{~K}$ provide good coverage of the peak of the thermal dust emission. Herschel observations thus enable the most accurate determination of the obscured star formation within GRBHs.

Our GRBH Herschel observations presented here, combined with the sample of GRBHs presented in Hunt et al. (2014, HPM14 from here on), in addition to GRBH 980425 (Michałowski et al. 2014) and GRBH 031203 (Symeonidis et al. 2014), provide a total sample of 23 GRBHs observed with Herschel. The GRBHs in HPM14 hosted GRBs with a range in visual extinctions, and we combine this sample with the GRBHs presented in this paper to investigate a larger GRB afterglow and host galaxy parameter space. In Sect. 2 we summarise previous multi-wavelength observations taken of our GRBH sample, and we describe our Herschel observations and data reduction. In Sect. 3 we present the results from our spectral energy distribution (SED) data analysis, and we summarise the principle characteristic properties of our sample in Sect. 4 and compare them with the literature. Finally, in Sect. 5 we explore the implications of the Herschel GRBH detection rate on the origin of the GRB afterglow extinguishing dust. Our conclusions are summarised in Sect. 6. Throughout the paper, we assume a $\Omega_{\mathrm{m}}=0.3, \Omega_{\Lambda}=$ 0.7 cosmology, with Hubble constant $H_{0}=70 \mathrm{~km} \mathrm{~s}^{-1} \mathrm{Mpc}^{-1}$.

\section{The sample}

Our host galaxy sample was built up using two selection criteria, both aimed at targeting those host galaxies with significant amounts of dust, and thus with a high chance of being detected with Herschel. Firstly, we selected the host galaxies of those GRBs with significantly dust-extinguished afterglows (i.e. $A_{V, \mathrm{GRB}}>1 \mathrm{mag}$ ) that were at $z<1.5$ and that had been observed at submillimetre (submm) wavelengths as part of our programme with the LABOCA instrument (Siringo et al. 2009) on APEX (Güsten et al. 2006). By limiting our sample to redshifts $z<1.5$ we increase our chance of a detection due to the Herschel sensitivity limit, and the additional submm data provides constraints on the dust emission at wavelengths longward of the thermal emission peak. At the time of the submission of the Herschel proposal, this gave us a sample of three GRBHs. In addition to this sample of host galaxies, we also included those GRBHs for which dust emission had already been detected at $850 \mu \mathrm{m}$ with SCUBA on James Clerk Maxwell Telescope (Holland et al. 1999), which increased the sample by a further two host galaxies. All five host galaxies in our sample have been well observed at optical and NIR wavelengths, as well as with the Spitzer Space Telescope.

\subsection{Afterglow parameters}

The five GRB host galaxies in our sample are listed in Table 1 together with some properties related to the GRB optical and $\mathrm{X}$-ray afterglow. In addition to the afterglow visual extinction, $A_{V, \mathrm{GRB}}$, we also list the afterglow optical-to-X-ray spectral index, $\beta_{\text {OX }}$ (Jakobsson et al. 2004), which provides a diagnostic tool for identifying GRBs with optical afterglows dimmer than expected by GRB synchrotron emission theory. In those cases where the GRB redshift is known to be $z<4$ (i.e. the optical afterglow is not significantly absorbed by neutral hydrogen within the intergalactic medium), a spectral index $\beta_{\mathrm{OX}}<0.5$ is an indicator of a dust-extinguished afterglow. The spectral index $\beta_{\mathrm{OX}}$ is much easier to measure than $A_{V, \mathrm{GRB}}{ }^{1}$, although it does not quantify the amount of dust along the GRB sight-line. We discuss further the merits of $A_{V, \mathrm{GRB}}$ and $\beta_{\mathrm{OX}}$ at identifying GRB host galaxies with appreciable levels of dust in Sect. 5.

All Swift GRBs (GRB 070306, GRB 081109, GRB 090926B) had their afterglows clearly detected at optical and NIR wavelengths with ground-based facilities, and at X-ray wavelengths with the X-ray telescope onboard Swift (Burrows et al. 2005). The GRB afterglow is typically well explained by synchrotron emission, and thus the extinction and absorption from dust and gas of the intrinsically simple

1 Whereas an X-ray afterglow flux and $R$-band afterglow flux limit is sufficient to determine $\beta_{\mathrm{OX}}$, an accurate determination of $A_{V, \mathrm{GRB}}$ requires the GRB afterglow to be detected in the X-ray and at least four optical or NIR filters (Schady et al. 2012), with NIR afterglow measurements becoming increasingly important at larger $A_{V, \mathrm{GRB}}$. 
Table 1. GRB afterglow properties.

\begin{tabular}{|c|c|c|c|c|c|c|c|}
\hline GRB & Redshift & \multicolumn{2}{|c|}{$(\mathrm{J} 2000)^{\ddagger}$} & $\begin{array}{l}\text { Offset }^{\dagger} \\
(\operatorname{arcsec})\end{array}$ & $\begin{array}{c}A_{V, \mathrm{GRB}} \\
\text { (mag) }\end{array}$ & $\beta_{\mathrm{OX}}^{\S}$ & $\begin{array}{c}N_{\mathrm{H}, \mathrm{X}} \\
10^{22} \mathrm{~cm}^{-2}\end{array}$ \\
\hline 000210 & $0.846^{1}$ & 01:59:15.58 & $-40: 39: 33.02^{6}$ & $<0^{\prime} 2^{11}$ & - & $<0.54$ & $0.17 \pm 0.04^{17}$ \\
\hline 000418 & $1.118^{2}$ & $12: 25: 19.30$ & $+20: 06: 11.6^{7}$ & $<0.4^{\prime 2}$ & $0.4-0.9^{14,15}$ & - & - \\
\hline 070306 & $1.496^{3}$ & 09:52:23.31 & $+10: 28: 55.26^{8}$ & $<0 ! 2^{13}$ & $5.5_{-1.0}^{+1.2,16}$ & $<-0.08$ & $2.5_{-0.2}^{+0.3,16}$ \\
\hline 081109 & $0.979^{4}$ & 22:03:09.72 & $-54: 42: 39.5^{9}$ & $<0.2^{\prime 3}$ & $3.4_{-0.3}^{+0.4,16}$ & $<0.3$ & $1.1_{-0.1}^{+0.1,16}$ \\
\hline 090926B & $1.243^{5}$ & 03:05:13.94 & $-39: 00: 22.2^{10}$ & $<0^{\prime} \cdot 6^{13}$ & $1.4_{-0.6}^{+1.1,16}$ & $<0.4$ & $2.2_{-0.5}^{+0.5,16}$ \\
\hline
\end{tabular}

Notes. ${ }^{(\ddagger)}$ All positions are from the optical afterglow with the exception of GRB 000210, which corresponds to the X-ray afterglow position. (†) Relative offset between the GRB afterglow position and the centre of the host galaxy. $\left.{ }^{(}\right)$GRB afterglow optical-to-X-ray flux spectral index as defined in Jakobsson et al. (2004). Those GRBs with afterglow spectral index $\beta_{\mathrm{OX}}<0.5$ have lower optical-to-X-ray flux ratios than predicted by the standard afterglow synchrotron theory, and as such are referred to as "dark".

References. ${ }^{(1)}$ Piro et al. (2002); (2) Bloom et al. (2003); (3) Jaunsen et al. (2008); ${ }^{(4)}$ Krühler et al. (2011); (5) Fynbo et al. (2009); (6) Piro et al. (2002); ${ }^{(7)}$ Klose et al. (2000); ${ }^{(8)}$ Rol et al. (2007); ${ }^{(9)}$ D'Avanzo et al. (2008); ${ }^{(10)}$ Malesani et al. (2009); ${ }^{(11)}$ This work; ${ }^{(12)}$ Berger et al. (2003); (13) Krühler et al. (2011); ${ }^{(14)}$ Klose et al. (2000); ${ }^{(15)}$ Berger et al. (2001); ${ }^{(16)}$ Krühler et al. (2011); ${ }^{(17)}$ Piro et al. (2002).

afterglow spectrum (power law or broken power law) can be well measured from the afterglow SED, in particular when afterglow data at the mostly unattenuated NIR and X-ray $(\gtrsim 2 \mathrm{keV}$ ) wavebands are available (Schady et al. 2007; Greiner et al. 2011). For each Swift GRB, $A_{V, \text { GRB }}$ is thus well measured. Note that this is the host galaxy visual extinction along the GRB line of sight, and thus does not necessarily reflect the galaxy-averaged visual extinction.

No optical or NIR afterglow was detected for GRB 000210 $(z=0.846)$, although the derived deep afterglow upper limits $(R>23.5$ at $12.4 \mathrm{~h}$ after the GRB trigger) and relatively bright X-ray afterglow suggests that the optical/NIR afterglow was extinguished by significant amounts of dust along the line of sight (Piro et al. 2002). GRB 000418 was detected at optical and NIR wavelengths. However, the lack of an X-ray detection, and the relatively late and thus dim optical/NIR afterglow detection provided only a fairly crude measure of the visual extinction in the range $A_{V, \mathrm{GRB}}=0.4-0.9 \mathrm{mag}$ (Klose et al. 2000; Berger et al. 2001). Nevertheless, $A_{V, \mathrm{GRB}}=0.4 \mathrm{mag}$ would place GRB 000418 within the $25 \%$ most dust-extinguished GRB afterglows at any redshift.

In the following subsections we provide a brief description of the host galaxy data available for our sample, all of which were taken once the afterglow had faded below the sensitivity limit of the instruments.

\subsection{Host galaxy observations}

\subsubsection{Optical and near-infrared data}

All GRBHs in our sample have a large quantity of published optical and NIR data, as well as er data, which in the case of GRB 090926B have not been published, and we thus analysed the data ourselves. We list all optical and NIR data used in our SED analysis in Tables A.1-A.4, together with corresponding references.

\subsubsection{Submillimetre data}

Prior to 2010, around 25 GRB host galaxies had been observed at $850 \mu \mathrm{m}$ with SCUBA (Berger et al. 2003; Tanvir et al. 2004), of which only the hosts of GRB 000210, GRB 000418, and GRB 010222 were reported to have detections at the $3 \sigma$ level. Since then, the submm emission thought to have come from the host galaxy of GRB 010222 has been put in doubt, and is instead likely to originate from an unrelated, nearby source (Michałowski et al. 2008). We observed the remaining three host galaxies in our sample at $850 \mu \mathrm{m}$ with the LABOCA instrument on APEX over two consecutive semesters during MPG guaranteed time (PI: Greiner). The observations were taken between April and August in 2011, and amounted to an average on-source integration time of $1.5 \mathrm{~h}$ per source. All submm flux measurements are listed in Table A.5.

\subsubsection{Herschel observations}

Our sample of GRB host galaxies was observed with the Herschel Space Observatory at $100 \mu \mathrm{m}$ and $160 \mu \mathrm{m}$ using the PACS small-scan map mode $\left(20^{\prime \prime} / \mathrm{s}\right)$ (Poglitsch et al. 2010), and all three SPIRE $250 \mu \mathrm{m}, 350 \mu \mathrm{m}$ and $500 \mu \mathrm{m}$ bands using the small-map mode (Griffin et al. 2010). Our measurements reached typical $1 \sigma$ sensitivities of $1.0 \mathrm{mJy}, 2 \mathrm{mJy}, 8 \mathrm{mJy}, 10 \mathrm{mJy}$ and $20 \mathrm{mJy}$ at $100 \mu \mathrm{m}, 160 \mu \mathrm{m}, 250 \mu \mathrm{m}, 350 \mu \mathrm{m}$ and $500 \mu \mathrm{m}$, respectively (included instrumental and confusion noise). The PACS observations of GRBH 000210 were shorter than for the rest of the sample, and thus reached $1 \sigma$ sensitivities of about $2 \mathrm{mJy}$ and $4 \mathrm{mJy}$ at $100 \mu \mathrm{m}$ and $160 \mu \mathrm{m}$, respectively. In the case of the SPIRE observations the sensitivities were predominantly determined by the confusion limit, which is $2-3$ times larger than the instrument point source sensitivity. The PACS and SPIRE beam FWHM sizes are 6.8", 11.4", 17.6", 23.9" and $35.2^{\prime \prime}$ at $100 \mu \mathrm{m}, 160 \mu \mathrm{m}, 250 \mu \mathrm{m}, 350 \mu \mathrm{m}$ and $500 \mu \mathrm{m}$, respectively. Herschel images for each GRBH in the bluest band observed are shown in Figs. 1 and 2.

Given the large uncertainty associated with predicting the FIR host galaxy flux from the UV through to mid-IR spectrum, we chose to initially observe our sample with SPIRE, in order to get good coverage of the thermal dust emission component. For the redshift range of our GRBH sample, we expected the dust emission to peak at $\sim 200 \mu \mathrm{m}$ in the observer frame. The three SPIRE bands just redward of this thus allow relatively accurate determination of the shape of the thermal dust component. Depending on the flux that we measured within the SPIRE bands, we then re-adjusted the exposure times used for our PACS observations within the total observing time available for our programme. We did not detect any of the four GRB hosts initially observed with SPIRE (see Table 2). In the case of GRBH 070306, which had a full-visibility window fairly late in the OT2 observing period, we therefore chose to only observe in 
Table 2. Herschel observations.

\begin{tabular}{lcclcc}
\hline \hline GRB Host & Instrument & Date/OD & OBSID & Duration (s) & On-source time (s) \\
\hline 000210 & SPIRE & $2012-05-11 / 1093$ & 1342245552 & 1135 & 296 \\
& PACS & $2012-12-11 / 1308$ & 1342256975,76 & $2 \times 840$ & $2 \times 270$ \\
000418 & SPIRE & $2012-07-12 / 1156$ & 1342247974 & 1135 & 296 \\
070306 & PACS & $2012-10-30 / 1266$ & 1342254142,43 & $2 \times 2250$ & $2 \times 720$ \\
081119 & SPIRE & $2012-05-10 / 1092$ & 1342245514 & 1135 & 296 \\
& PACS & $2012-10-16 / 1251$ & 1342253509,10 & $2 \times 2250$ & $2 \times 720$ \\
$090926 B$ & SPIRE & $2012-03-01 / 1022$ & 1342239863 & 1135 & 296 \\
& PACS & $2013-01-05 / 1333$ & 1342258541,42 & $2 \times 2250$ & $2 \times 720$ \\
\hline
\end{tabular}

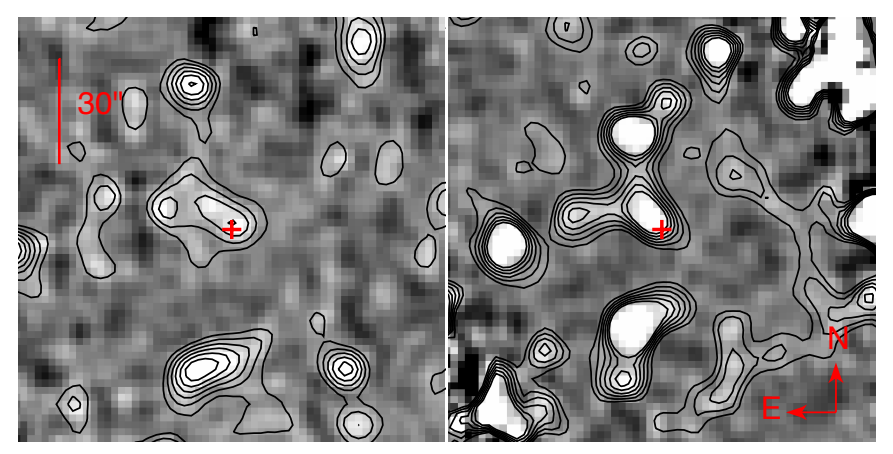

Fig. 1. Herschel/PACS $100 \mu \mathrm{m}$ and $160 \mu \mathrm{m}$ images of GRBH 070306. The images are $2^{\prime} \times 2^{\prime}$ and $3^{\prime} \times 3^{\prime}$, respectively, centred on the GRB afterglow position (red cross), and have been smoothed using a Gaussian with $\sigma$ two times the pixel scale. The image is displayed with a linear greyscale ranging from $-1 \mathrm{mJy} \mathrm{pix}^{-1}$ (black) to $+1 \mathrm{mJy} \mathrm{pix}^{-1}$ (white), and contour levels from $1 \sigma$ to $6 \sigma$ are over plotted in back. The spatial scale is indicated on the top left.

PACS, which although provides coverage over a smaller wavelength range ${ }^{2}$, is more sensitive than SPIRE. Finally, in the case of GRBH 000418, our broadband SED template fits to the optical/NIR data and SPIRE upper limits resulted in estimated flux densities that were below the sensitivity limit of PACS. We therefore did not observe GRBH 000418 with PACS, instead choosing to redistribute this time within our PACS observations. Details of the Herschel observations are listed in Table 2.

\subsubsection{Herschel data reduction}

Data reduction in PACS and SPIRE was performed using the Herschel Interactive Processing Environment (HIPE v10.0.0; Ott 2010). The PACS data were reduced using the "deep survey point-source" script within HIPE. We used pixel sizes of $2^{\prime \prime}$ and $3^{\prime \prime}$ for PACS $100 \mu \mathrm{m}$ and $160 \mu \mathrm{m}$, and $6^{\prime \prime}, 10^{\prime \prime}$ and $14^{\prime \prime}$ for SPIRE $200 \mu \mathrm{m}, 350 \mu \mathrm{m}$ and $500 \mu \mathrm{m}$, respectively. The individual PACS scans were processed with a high pass filter to remove $1 / f$ noise and thermal drifts in the PACS bolometers. We used a running box median filter with a half-width of 31 frames $\left(62^{\prime \prime}\right)$ at $100 \mu \mathrm{m}$ and 51 frames $\left(102^{\prime \prime}\right)$ at $160 \mu \mathrm{m}$. This choice of the high-pass filter radius allows us to optimise the $1 / \mathrm{f}$ noise subtraction, thus reducing the final map noise without degrading the PACS PSF. We initially stacked the cross-scans to create a mask from this deeper image, and the individual cross-scans were then re-reduced and stacked, this time using our newly created mask.

\footnotetext{
2 Although the PACS photometer offers three bands $(70 \mu \mathrm{m}, 100 \mu \mathrm{m}$ and $160 \mu \mathrm{m}$ ), the $160 \mu \mathrm{m}$ filter can only be used with one of the other two filters simultaneously. For our observations we opted for the $100 / 160 \mu$ m combination.
}

Table 3. Herschel PACS and SPIRE photometric measurements.

\begin{tabular}{lccccc}
\hline \hline \multicolumn{7}{c}{ Flux density (mJy) } \\
\hline GRB Host & $100 \mu \mathrm{m}$ & $160 \mu \mathrm{m}$ & $250 \mu \mathrm{m}$ & $350 \mu \mathrm{m}$ & $500 \mu \mathrm{m}$ \\
\hline 000210 & $<5.1$ & $<11.1$ & $<24.0$ & $<36.4$ & $<38.6$ \\
000418 & - & - & $<22.5$ & $<35.8$ & $<33.4$ \\
070306 & $4.4_{-1.0}^{+1.0}$ & $6.2_{-1.2}^{+1.2}$ & - & - & - \\
081109 & $<4.0$ & $<6.6$ & $<26.8$ & $<27.5$ & $<18.8$ \\
$090926 \mathrm{~B}$ & $<3.2$ & $<5.3$ & $<22.0$ & $<23.2$ & $<26.3$ \\
\hline
\end{tabular}

Notes. Upper limits are given at $3 \sigma$ confidence.

Finally, we corrected the astrometry in our final, stacked, images using the aspect solution from the bluest Spitzer Infrared Array Camera observations available for each field. These corrections were typically of the order of $2^{\prime \prime}$ at $100 \mu \mathrm{m}$ at $3^{\prime \prime}$ at $160 \mu \mathrm{m}$.

Fluxes were measured at the source location using the HIPE internal aperture photometry routines and checked with IDLbased procedures. For the SPIRE images, aperture radii of $22^{\prime \prime}$, $30^{\prime \prime}$ and $42^{\prime \prime}$ were used at $250 \mu \mathrm{m}, 350 \mu \mathrm{m}$ and $500 \mu \mathrm{m}$, respectively, in line with SPIRE calibration guide lines (Pearson et al. 2013). For the PACS stacked images, we used radii of $5^{\prime \prime}$ and $7.5^{\prime \prime}$ at $100 \mu \mathrm{m}$ and $160 \mu \mathrm{m}$, respectively, in order to gain the highest signal-to-noise ratio for a point source (Pérez-González et al. 2010), applying a respective aperture correction of 1.9 and 2.0 (Balog et al. 2013). Colour corrections are of the order of unity, and we therefore neglect them (Poglitsch et al. 2010).

In the case of GRBH 070306, there is a source 10" north-east of the GRB host galaxy. In order to disentangle the flux emission from the host galaxy of GRB 070306 and the nearby source, we used the GALFIT package (Peng et al. 2002) to measure the flux from the two sources simultaneously based on the observed Herschel PSF. At $160 \mu \mathrm{m}$ the FWHM beam size $\left(11.4^{\prime \prime}\right)$ does not allow the GRB host galaxy and neighbouring source to be resolved, and we therefore fixed the position of the two sources to the $100 \mu \mathrm{m}$ best-fit positions found with GALFIT. The $100 \mu \mathrm{m}$ source positions were within $0.3^{\prime \prime}$ and $1.6^{\prime \prime}$ of the GRBH and nearby source centroid positions, respectively, in the $4.5 \mu \mathrm{m}$ Spitzer image.

In all cases, the rms sky noise was calculated from the standard deviation of 100 circular apertures of the same size as the source extraction region, randomly placed around the source position, within high-coverage regions of the image. This provides an accurate measure of the sky background and includes correlated noise (see Sect. 4.2.2 in Balog et al. 2013). Our SPIRE and PACS flux measurements are listed in Table 3.

\section{Data analysis}

Given that by selection, all the host galaxies in our sample showed evidence of dust along the GRB line of sight, the lack 


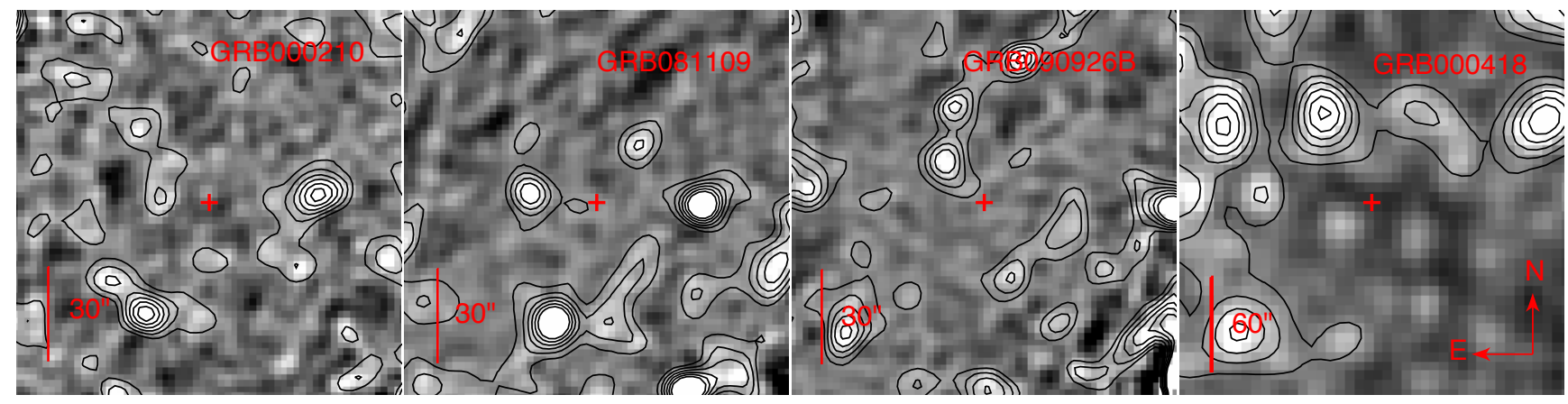

Fig. 2. Herschel/PACS $100 \mu \mathrm{m}$ images of GRBH 000210, GRBH 081109 and GRBH 090926B (first three panels), and Herschel/SPIRE 250 $\mu \mathrm{m}$ image of GRBH 000418 (furthest right). The PACS and SPIRE images are $2^{\prime} \times 2^{\prime}$ and $4^{\prime} \times 4^{\prime}$, respectively, centred on the GRB afterglow position (red cross), and have been smoothed using a Gaussian with $\sigma$ two times the pixel scale. The images are displayed with a linear greyscale ranging from $-1 \mathrm{mJy} \mathrm{pix}^{-1}$ (black) to $+1 \mathrm{mJy} \mathrm{pix}^{-1}$ (white), and contour levels from $1 \sigma$ to $6 \sigma$ are over plotted in back.

of a Herschel detection in four out of five cases may imply that the dust mass within these galaxies is generally low, and concentrated in a few discrete locations within the galaxy. In the following section we describe our analysis and provide our bestfit results from our SED fits.

\subsection{Spectral energy distributions}

For each GRB host galaxy in our sample we combined all optical through to submm flux density measurements to create a broadband SED. Most of our sample SEDs have no data between $10 \mu \mathrm{m}$ and $100 \mu \mathrm{m}^{3}$, and there are few detections redward of $25 \mu \mathrm{m}^{4}$ (see Fig. 3). We therefore chose to place constraints on the host galaxy dust properties by fitting just the FIR and submm data separately with a modified blackbody (MBB). The emissivity index was set to $\beta=1.5$ and the grain absorption cross section per unit mass was set to $\kappa_{\mathrm{abs}}=3.4 \mathrm{~cm}^{2} \mathrm{~g}^{-1}$ at $250 \mu \mathrm{m}$ rest frame (Bianchi 2013). These values are consistent with the thermal emission measured in other star forming galaxies (i.e. $\beta=1.5-2$, Dunne \& Eales 2001; Dale et al. 2012; Casey 2012). In the case of GRBH 070306, the dust temperature was constrained by the two PACS detections, and thus this parameter was left free to vary. For the remaining four GRBHs, the temperature was fixed to a typical value of $35 \mathrm{~K}$ (Skibba et al. 2011; Magnelli et al. 2012a; Rémy-Ruyer et al. 2013; Sklias et al. 2014), although in the case of GRBH 000210 and GRBH 000418, the SCUBA detections provided further constraint on the dust temperature, and we found that temperatures of $30 \mathrm{~K}$ and $40 \mathrm{~K}$, respectively were more compatible with the data (see Sects. 3.2.2 and 3.2.2 for details).

From our SED fits, we determined the upper limit on the dust mass allowed by our data, which was mostly constrained by the bluest PACS data at $100 \mu \mathrm{m}$, and we used the total luminosity $(8-1000 \mu \mathrm{m})$ measured from our best-fit MBB model to estimate the FIR SFR using the prescription from Kennicutt (1998). In all cases, the FIR-derived SFR or upper-limits are higher than the optically derived SFRs by at least $30 \%$, suggesting that, at least in the case of GRBH 070306, there is some obscured star formation (see Table 4).

\footnotetext{
3 Only GRBH 000210 was observed within this wavelength range, at $24 \mu \mathrm{m}$ with Spitzer.

4 Two detections of GRBH 070306 with PACS at $100 \mu \mathrm{m}$ and $160 \mu \mathrm{m}$, and two tenuous detections of GRBH 000210 and GRBH 000418 at $850 \mu \mathrm{m}$ with SCUBA.
}

We repeated our fits for an emissivity index $\beta=2$, and found that this has a weak effect on our total IR luminosity, but it typically decreases the dust mass by 0.3 dex. A change in the blackbody temperature has a much stronger effect, with the dust mass varying over two orders of magnitude for dust temperatures in the range $20-50 \mathrm{~K}$, and the SFR varying by $\sim 30 \%$. This uncertainty is most relevant for GRBH 0811109 and GRBH 090926B, for which there was no detected FIR or submm emission with which to constrain the dust temperature. However, both these host galaxies have relatively high un-obscured star formation rates (see Table 4), and thus would be expected to have relatively high dust temperatures ( $>35 \mathrm{~K}$ ) (Hunt et al. 2014; Sklias et al. 2014). The dust mass is inversely related to the dust temperature, and we therefore consider our dust mass upper limits for both GRBH 081109 and GRBH 090926B to be fairly conservative, in that the true dust mass is likely to be discernibly lower than our stated limit. A more important source of error is in our assumption of a single-temperature MBB, which does not take into account colder dust that can make up $50 \%$ of the total dust mass (e.g. Dale et al. 2012; Magdis et al. 2012, 2013). For those galaxies without a submm detection, for which the emission from a cold-dust component cannot be constrained, we increased our dust mass errors to 0.3 dex to account for the uncertainty in the dust temperature. The broadband SEDs for each GRB host in our sample are shown in Fig. 3 together with the MBB model.

Data below $25 \mu \mathrm{m}$ were fitted with the spectral template fitting package LePHARE (Arnouts et al. 1999; Ilbert et al. 2006), which is a population-synthesis-based fitting procedure. We used the Bruzual \& Charlot (2003) galaxy templates, which include emission lines and prescribed reddening and parameters therein, and we assumed a Chabrier (2003) IMF.

\subsection{Comparison with the literature}

\subsubsection{Stellar mass and SFR}

After correcting for differences in the assumed IMF in this work and in other publications (i.e. Michałowski et al. (2008) assumed a Salpeter (1955) IMF and Savaglio et al. (2009; SGL09 from here on) assumed a Baldry \& Glazebrook (2003) IMF), our stellar masses are in agreement (at $1 \sigma$ ) with other reported values (i.e. SGL09; Krühler et al. 2011; PLT13; HPM14), with the exception of the higher $M_{*}$ found by Michałowski et al. (2008) for GRBHs 000210 and 000418 . After converting to a Chabrier (2003) IMF, the $M_{*}$ in Michałowski et al. (2008) is a factor of 

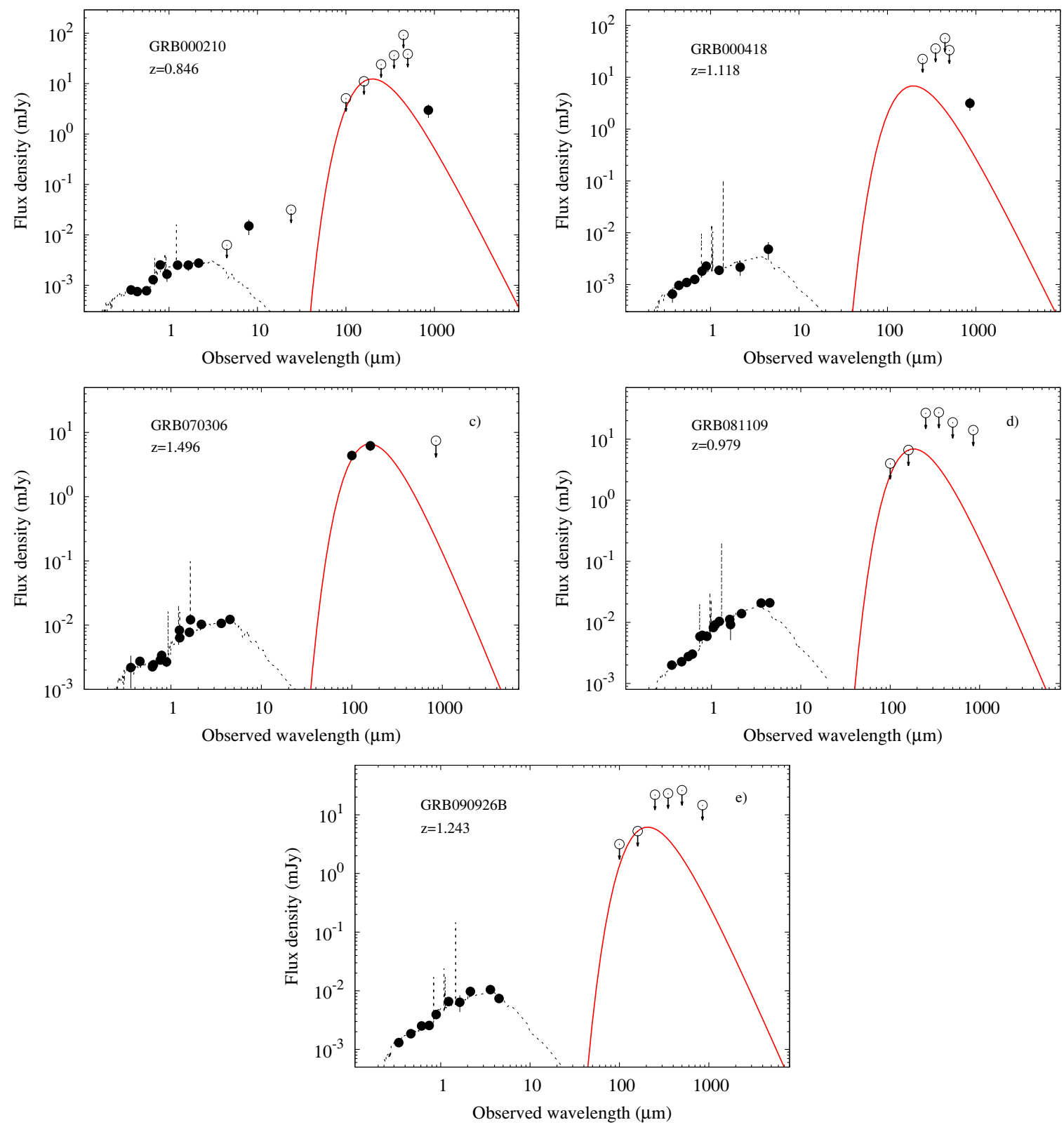

Fig. 3. GRB host galaxy optical through to submm spectral energy distributions for our sample of five GRBHs observed with Herschel. Detections are plotted as filled symbols, and open circles represent $3 \sigma$ upper limits. The lePHARE best-fit template galaxy models fitted to the optical to mid-IR data are shown (dashed black line), as well as our modified blackbody fits to the FIR and submm data (red solid line) (see Table 4 for details). In those cases where the host galaxy is not detected with Herschel, the modified blackbody fits are used to determine the upper limit on the corresponding dust mass and SFR.

3 and 14 higher than ours, respectively, which may be the result of differences in the star formation histories and stellar populations assumed in the modelling (Michałowski et al. 2012a, see Sect. 4). The SED GRASIL fitting software package (Silva et al. 1998) used by Michałowski et al. (2008) accounts for the stellar mass from a starburst as well as from continuous star formation, and can increase the best-fit stellar mass by up to 0.4 dex (relative to optical/NIR-only SED fits). The inclusion of FIR data used in Michałowski et al. (2008) may also increase $M_{*}$ if the best-fit templates include a fully dust-obscured stellar population (e.g. Lo Faro et al. 2013).

There is greater scatter within the literature amongst the optically derived SFRs. This is related to the uncertainty in the dust correction, which can vary by a factor of a few. Our SFR(FIR) for GRBH 070306 is consistent with HPM14, but our values for GRBHs 000210 and 000418 differ from the results in Michałowski et al. (2008) by a factor of 4 and 2, respectively. We comment on the possible reasons for this below.

\subsubsection{Dust mass and temperature}

\section{GRBH 000210}

This galaxy had a reported $850 \mu \mathrm{m} 3 \sigma$ detection (Berger et al. 2003; Tanvir et al. 2004), with $\mathrm{S}_{850 \mu \mathrm{m}}=3.0 \pm 0.9 \mathrm{mJy}$. A MBB with $T=35 \mathrm{~K}$ scaled to our Herschel limits underestimates the thermal emission at $850 \mu \mathrm{m}$ by one to two orders of magnitude for an emissivity index $\beta=1.5-2$. A MBB with a colder dust temperature of $T=30 \mathrm{~K}$ is in closer agreement to the Herschel and SCUBA observations, but only just at the $3 \sigma$ level (Fig. 3a, 
Table 4. GRB host galaxy properties taken from the literature and based on UV, optical, near- and mid-IR data, as well as the dust mass and temperature derived in this paper from modified blackbody fits to our Herschel data.

\begin{tabular}{lcccccccc}
\hline \hline $\begin{array}{l}\text { GRB } \\
\text { Host }\end{array}$ & $\begin{array}{c}E(B-V)^{a} \\
(\mathrm{mag})\end{array}$ & {$[\mathrm{O} / \mathrm{H}]^{b}$} & $\begin{array}{c}S F R(\mathrm{H} \alpha / \mathrm{OII})^{c} \\
\left(M_{\odot} \mathrm{yr}^{-1}\right)\end{array}$ & $\begin{array}{c}S F R(\mathrm{FIR}) \\
\left(M_{\odot} \mathrm{yr}^{-1}\right)\end{array}$ & $\begin{array}{c}\log \left[M_{*}\right] \\
\left(M_{\odot}\right)\end{array}$ & $\begin{array}{c}A_{V}{ }^{d} \\
(\mathrm{mag})\end{array}$ & $\begin{array}{c}T_{\mathrm{d}}{ }^{e} \\
(\mathrm{~K})\end{array}$ & $\begin{array}{c}\log \left[M_{\mathrm{d}}\right]^{f} \\
\left(M_{\odot}\right)\end{array}$ \\
\hline 000210 & 0.029 & $8.3 \pm 0.1$ & $1.1_{-0.1}^{+0.1}$ & $<37$ & $9.23_{-0.08}^{+0.12}$ & 0.0 & 30 & $<8.6$ \\
& & $8.7 \pm 0.1$ & & & & & & \\
000418 & 0.033 & $8.1 \pm 0.2$ & $26_{-1.4}^{+1.2}$ & $<40$ & $8.87_{-0.10}^{+0.15}$ & 1.3 & 40 & $<8.3$ \\
& & $8.8 \pm 0.2$ & & & & & & \\
070306 & 0.024 & $8.4 \pm 0.1$ & $61_{-10}^{+10}$ & $101 \pm 21$ & $10.34_{-0.29}^{+0.09}$ & 0.4 & $51 \pm 0.2$ & $7.9 \pm 0.3$ \\
081109 & 0.017 & $8.8 \pm 0.1$ & $24_{-9}^{+14}$ & $<32$ & $9.93_{-0.04}^{+0.06}$ & 1.3 & 35 & $<8.5$ \\
$090926 \mathrm{~B}$ & 0.020 & $8.2 \pm 0.2$ & $12_{-6}^{+12}$ & $<45$ & $9.76_{-0.05}^{+0.07}$ & 1.0 & 35 & $<8.7$ \\
\hline
\end{tabular}

Notes. ${ }^{(a)}$ Galactic dust reddening in magnitudes from the map of Schlafly \& Finkbeiner (2011). ${ }^{(b)}$ Metallicities in the case of GRBH 000210 , 000418 and 070306 (Piranomonte et al., in prep.; Vergani et al., in prep.) were derived using the $R_{23}$ ratio and applying the calibration from Kobulnicky \& Kewley (2004). The N II emission line required to distinguish between the $R_{23}$ lower and upper branches was not detected in the spectra of GRBH 000210 and 000418, and we therefore report both solutions. Metallicities for GRBH 081109 and 090926B (Krühler et al., in prep.) were derived using the methods from Nagao et al. (2006). For a detailed description on the differences between the individual strong line diagnostics, see Kewley \& Ellison (2008). ${ }^{(c)}$ SFRs derived from the O II line for GRBH 000418 (Piranomonte et al., in prep.), and from the H $\alpha$ line for the other four GRBHs (Piranomonte et al., in prep.; Krühler et al., in prep.; Vergani et al., in prep.), based on the formulation described in Kennicutt (1998), but converted to a Chabrier IMF (Chabrier 2003). All have been corrected for host galaxy dust extinction derived from the Balmer decrement, with the exception of GRBH 000418, for which we used the average visual extinction given in Col. 7, and assuming the Calzetti dust attenuation law (Calzetti et al. 2000). ${ }^{(d)}$ The quoted value corresponds to the $A_{V}$ of the best-fit galaxy template. ${ }^{(e)}$ Fixed temperature of the MBB scaled to the data, apart from in the case of GRBH 070306, where it was left as a free parameter in the SED fit. ${ }^{(f)} 3 \sigma$ upper limit or best-fit dust-mass resulting from a blackbody scaled to the data with emissivity index $\beta=1.5$ and temperature as given in Col. 8 .

red line). Although a MBB peaking at even colder dust temperatures could theoretically be scaled up to the SCUBA flux measurement without exceeding the Herschel upper limits, the resulting dust mass would lead to unfeasibly high dust-to-stellar mass ratios. Some SMGs can have dust-to-stellar mass ratios as high as 0.3 (Michałowski et al. 2010), but these are extreme cases and more typical values are closer to $\sim 0.1$. A dust temperature of $T=30 \mathrm{~K}$ and dust mass $\log \left[M_{\mathrm{d}} / M_{\odot}\right]=8.6$ therefore provides the greatest consistency with the data.

Michałowski et al. (2008) found the host galaxy SED to be best-fit by a higher effective temperature of $45 \mathrm{~K}$. However, these fits lacked our Herschel data, which place an upper limit on the amplitude of the thermal dust emission. In our model, the smaller normalisation of a MBB imposed by the Herschel upper limits (and thus lower $850 \mu \mathrm{m}$ emission) is counter-acted by a decrease in the dust temperature. The Herschel upper limits similarly result in a lower SFR(FIR) than in Michałowski et al. (2008). The marginal consistency between our model and the submm flux measurement places the SCUBA detection somewhat in doubt, and in Table 4 we thus give this dust mass as an upper limit.

\section{GRBH 000418}

This galaxy was also detected at the $3 \sigma$ level with SCUBA, with a flux density $\mathrm{S}_{850 \mu \mathrm{m}}=3.2 \pm 0.9 \mathrm{mJy}$ (Berger et al. 2003; Tanvir et al. 2004). The dust mass upper limit derived from a $T=35 \mathrm{~K} \mathrm{MBB}$ fit to our Herschel data is only just consistent with the SCUBA detection at the $3 \sigma$ level. However, our bestfit dust mass of $\log \left[M_{\mathrm{d}} / M_{\odot}\right]=8.9$ is almost the same as the galaxy stellar mass, which as explained above, becomes difficult to explain physically. Applying a dust-to-gas ratio of 0.1 gives a considerably lower dust mass of $\log \left[M_{\mathrm{d}} / M_{\odot}\right]=8.3$. It is possible to remain within this dust mass upper limit and reproduce the SCUBA flux measurement (albeit only just within the $3 \sigma$ limit) by increasing the temperature of the warm component to $40 \mathrm{~K}$. When scaled to the PACS $100 \mu \mathrm{m}$ upper limit, a temperature any higher than $40 \mathrm{~K}$ would fail to reproduce the SCUBA flux measurement.
The effective temperature fitted by Michałowski et al. (2008) was $50 \mathrm{~K}$, and their SFR(FIR) was also higher than our upper limit, and this is similarly related to the additional data coverage blueward of $450 \mu \mathrm{m}$ provided by our Herschel PACS data. For a similar reason as in the case of GRBH 000210, we report the dust mass of $\log \left[M_{\mathrm{d}} / M_{\odot}\right]=8.3$ as an upper limit.

\section{GRBH 070306}

This is the only host galaxy from our sample that was detected with Herschel. Assuming a single MBB component, the best-fit dust temperature and mass are $T=51.2 \pm 0.1 \mathrm{~K}$ and $\log \left[M_{\mathrm{d}} / M_{\odot}\right]=7.9 \pm 0.3$.

This GRB host galaxy was also included in the sample of galaxies studied in HPM14, in which the optical through to radio host galaxy data were fitted simultaneously using the software package GRASIL. This treats the stellar light absorbed by dust, and the re-emitted dust emission at FIR and submm wavelengths in a self-consistent way, and includes emission from dust grains with a continuous distribution of temperatures, in addition to emission from mid-IR wavelengths from polycyclic aromatic hydrocarbons (PAHs). In addition to the different SED modelling used in this paper and in HPM14, the Herschel PACS photometry for GRBH 070306 also differed, in particular at $160 \mu \mathrm{m}$, although still consistent at the $2 \sigma$ level. The reason for this difference is most likely related to the methods used to remove the contamination from a nearby source (see Sect. 2.2.4). Whereas HPM14 applied aperture photometry within HIPE, we used the GALFIT software, as described in Sect. 2.2.4.

Despite the (small) differences in photometry, and the much simpler approach that we use in this paper to constrain the host galaxy dust properties, our best-fit dust mass $\left(\log \left[M_{\mathrm{d}} / M_{\odot}\right]=\right.$ $7.9 \pm 0.3)$ is consistent within $1 \sigma$ with the GRASIL best-fit value of $8.3 \pm 0.3$ in HPM14.

\section{GRBH 081109}

Emission at the position of this galaxy was detected with the LABOCA instrument on APEX at the $3 \sigma$ level, with a flux 

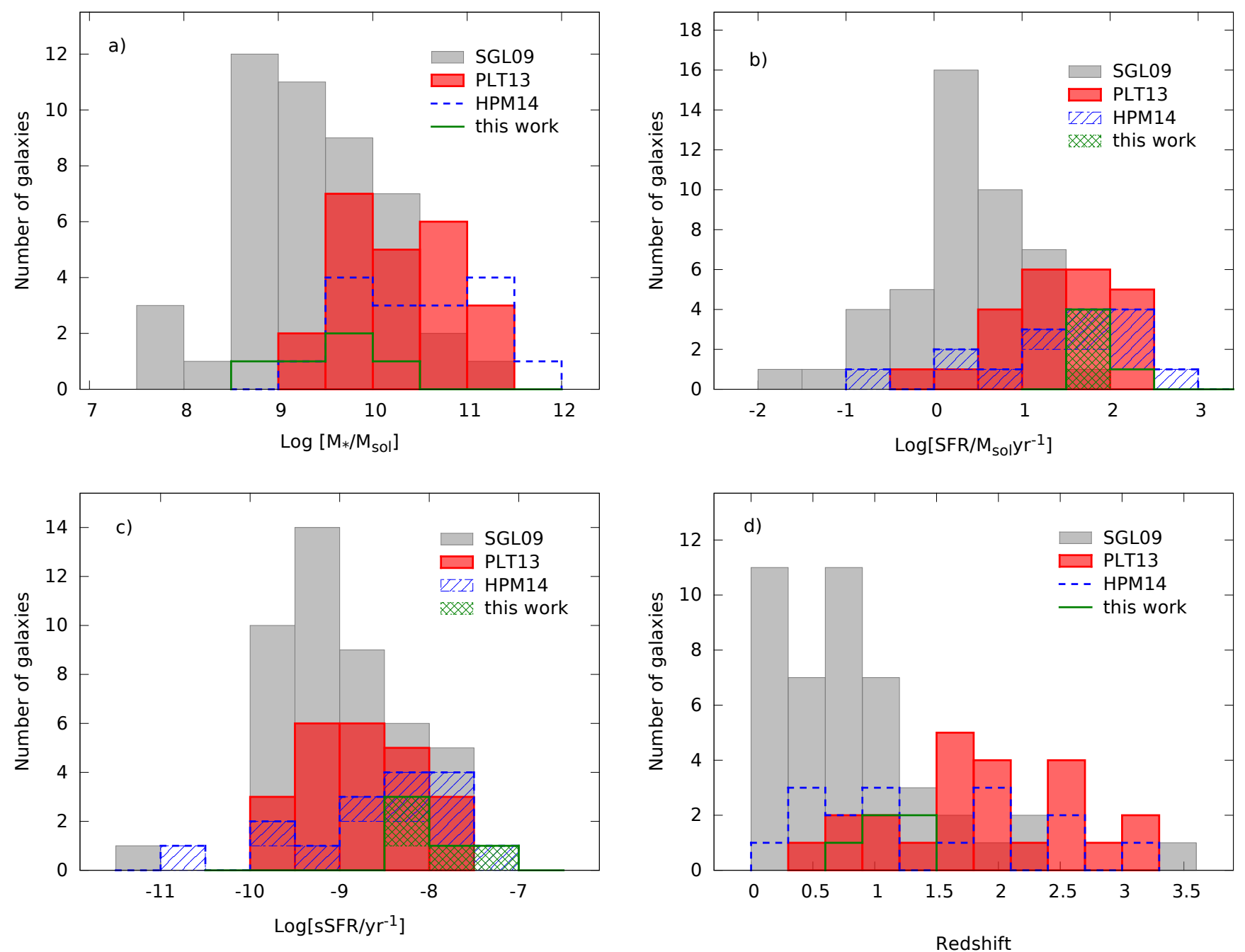

Fig. 4. Distribution of logarithmic $M_{*}$ (top left), SFR (top right) and SSFR (bottom left) as derived from SED analysis, and the redshift distribution (bottom right) from a number of GRB host galaxy samples. Outlined in solid green is the sample in this paper, in dashed blue is the sample of 16 GRBHs from HPM14 (not including GRBH 070306), in filled red is a sample of 23 host galaxies of heavily dust-extinguished GRBs $\left(A_{V, G R B}>1 \mathrm{mag}\right)$ from PLT13, and the filled grey histogram is a sample of 46 host galaxies of optically bright GRBs from SGL09. The samples from HPM14 and this work include SFR and SSFR upper limits, which are shown in hashed blue, and in a crisscross green pattern, respectively.

density $S_{870 \mu \mathrm{m}}=18.0 \pm 4.7 \mathrm{mJy}$. However, the non-detection of the host galaxy in both Herschel PACS and SPIRE bands would imply an unfeasibly low dust temperature $T<10 \mathrm{~K}$, indicating that the APEX detection was due to a spurious source or blending. Within the PACS and two bluest SPIRE images, there are three resolved sources within a $30^{\prime \prime}$ region around the GRB host position, all of which could have contributed to the flux density measured within the LABOCA $19^{\prime \prime}$ beam. We therefore conclude that the LABOCA detection was likely spurious or contaminated by an unrelated source, and determine a $3 \sigma$ upper limit on the host galaxy submm flux density of $14 \mathrm{mJy}$. Assuming an average dust temperature of $35 \mathrm{~K}$, the PACS upper limits then constrain the dust mass to $\log \left[M_{\mathrm{d}} / M_{\odot}\right]<8.5$.

\section{GRBH 090926B}

This galaxy was undetected in all PACS and SPIRE bands, as well as at $870 \mu \mathrm{m}$ with LABOCA, with a $3 \sigma$ upper limit of $\mathrm{S}_{870 \mu \mathrm{m}}<15 \mathrm{mJy}$. The greatest constraint to a MBB with temperature $T=35 \mathrm{~K}$ is provided by the $160 \mu \mathrm{m}$ PACS upper limit, yielding a dust-mass upper limit of $\log \left[M_{\mathrm{d}} / M_{\odot}\right]<8.7$.

\section{Summary of host galaxy properties}

Our sample was selected from the GRB optical afterglow properties, based on the expectation that dusty GRB sightlines are indicative of a host galaxy with a high dust mass. In order to see how these selection criteria affect the overall galaxy properties of the sample, we briefly summarise some of the characteristic properties of our host galaxy sample (see Table 4), and compare these to other GRB host galaxy samples.

In Fig. 4 we show the logarithmic distribution of $M_{*}$, SFR and sSFR for a number of GRB host samples, as well as the redshift distribution. The SGL09 sample (filled grey) was selected on the basis of optical and NIR detections, and is thus predominantly made up of host galaxies of GRBs with optically bright afterglows (i.e. small $A_{V, \mathrm{GRB}}$ at $z<1.5$ ). In contrast to this, the PLT13 sample (filled red) is composed of galaxies hosting heavily extinguished GRBs $\left(A_{V, \mathrm{GRB}}>1 \mathrm{mag}\right)$, and the sample of Herschel observed hosts from HPM14 (dashed blue outline) was selected on the basis of two or more host galaxy Spitzer detections $(\gtrsim 10 \mu \mathrm{Jy}$ at $3.6 \mu \mathrm{m}$ and $4.5 \mu \mathrm{m})$, with a preference for hosts of GRBs with $\beta_{\mathrm{OX}}<0.5$. 
Stellar masses and SFRs were derived from SED fits to the UV through to near- or mid-IR data in the SGL09 and PLT13 samples, and in the case of HPM14, FIR Herschel data were also included. In the HPM14 and our samples, SFRs were determined from the IR luminosity, which in most cases are thus upper limits. This is indicated in Figs. 4a and b with the blue hashed, and green crisscross pattern for the HPM14 and our samples, respectively.

Differences in the stellar population models and star formation history parameterisations used in the SED fits, as well as in the assumed dust attenuation, can introduce systematic differences in $M_{*}$ of up to 0.2-0.3 dex (Pozzetti et al. 2007; Kajisawa et al. 2009; Wuyts et al. 2009; Ilbert et al. 2010; Mitchell et al. 2013). In particular, SGL09 used an IMF from Baldry \& Glazebrook (2003), which gives a total stellar mass that is 0.2-0.3 dex higher than when assuming a Chabrier (2003) IMF, as was the case in the modelling of PLT13 and HPM14. Already without correcting for this, the stellar masses in SGL09 appear systematically lower than those from the other samples, and a Kolmogorov-Smirnoff (KS) test gives a $99.999 \%$ probability that the PLT13 and HPM14 samples come from a different parent population to the SGL09 sample.

Systematic differences in the SFR arise predominantly from the uncertainty in dust attenuation. The SFRs in SGL09 were derived from UV and optical photometry, thus making the level of dust-obscured star formation highly uncertain. The inclusion of Herschel observations in our sample and in HPM14 provide a better handle on the total SFR, and thus the SFRs in these two samples are typically higher than the SFRs in SGL09. The SFR remains uncertain for those GRBHs in HPM14 that were not detected with Herschel, and in these cases we therefore use the optically derived (dust corrected) SFRs when comparing the HPM14 and SGL09 sample SFRs. We find that the two SFR distributions are inconsistent with coming from the same parent population, with a null-hypothesis probability $P=0.01$, and $\mathrm{P}$ only increases to 0.02 when we correct the SFRs to the same IMF. The high SFRs in the PLT13 sample are more likely related to the selection criteria rather than greater sensitivity to the total SFR (host galaxies with $A_{V, \mathrm{GRB}}>1 \mathrm{mag}$ tend to be more massive and actively star forming). The sSFR in PLT13 and HPM14 are nevertheless consistent with the sSFR in SGL09 $(P=0.2$ and 0.5 , respectively).

For the majority of the samples considered here, it is unlikely that cosmic evolution is responsible for the differences observed in the $M_{*}$ and SFR, since the redshift distributions are fairly consistent between samples. Only the PLT13 sample shows a very different redshift distribution, with a mean redshift of $\langle z\rangle=1.8$, compared to mean redshifts of $\langle z\rangle=0.8,1.1$ and 1.1 for the SGL09, HPM14 and our sample.

\section{Discussion}

The initial aim of our observations had been to study in greater detail the dust properties of GRB host galaxies. In light of our single host galaxy detection, in the following section we look in closer detail at the differences in the galactic properties of those host galaxies that were and were not detected. In order to increase our statistics, we include the host galaxy sample from HMP14, and GRBH 980425 and GRBH 031203 from Michałowski et al. (2014) and Symeonidis et al. (2014) in our analysis. We emphasise that despite the fact that our sample is not complete, all GRBHs included are not "special" within the range of galaxy properties observed in GRBHs (e.g. SGL09, PLT13). Although our compiled sample is on average at the high end of the stellar-mass and SFR distribution (see Fig. 4), there is no compelling reason to believe that these galaxy properties should alter the relation between line of sight dust extinction and galaxy-whole dust emission. For our purposes, our combined sample thus provides a fair representation of dust properties of GRBHs.

\subsection{GRB line of sight versus galaxy-integrated properties}

From the combined sample of GRBHs from this paper, HPM14, Michałowski et al. (2014) and Symeonidis et al. (2014), a third were detected, and this fraction almost doubles when we only consider those hosts of GRBs with known $A_{V, \mathrm{GRB}}>1 \mathrm{mag}^{5}$. On the other hand, less than $40 \%$ of galaxies hosting so-called dark GRBs (i.e. $\beta_{\mathrm{OX}}<0.5$ ) were detected with Herschel. Although those GRBs in our sample with $A_{V, \mathrm{GRB}}>1 \mathrm{mag}$ are all classified as dark by the $\beta_{\mathrm{OX}}$ convention, the converse does not apply, and only $50 \%$ of GRBs classified as dark have $A_{V}$, GRB $>1$ mag. This is because although $\beta_{\mathrm{OX}}<0.5$ is suggestive of dust extinction, it does not necessarily imply significant amounts of dust, as is the case for $A_{V, \mathrm{GRB}}>1 \mathrm{mag}$. However, when we apply a more stringent upper limit on $\beta_{\mathrm{OX}}$ of $<0.4$ we then select all those GRBs with $A_{V, \mathrm{GRB}}>1 \mathrm{mag}$, as well as GRBH 970828 , for which no $A_{V, \mathrm{GRB}}$ information is available. When we only consider the hosts of those GRBs with a measured visual extinction $A_{V, \mathrm{GRB}}>1.5 \mathrm{mag}$ (equivalent to $\beta_{\mathrm{OX}}<0.3$ ) then the detection rate goes up further to three quarters of the sample.

The fairly high detection rate of hosts selected by their dust-extinguished GRBs implies that the extinguishing dust lies predominantly within the host galaxy ISM, rather than within discrete, dense clouds. If the afterglow extinction arose predominantly from dust within a molecular cloud, either related to or independent of the GRB natal region, then we would expect to have detected a similar number of galaxies with low and high $A_{V, \mathrm{GRB}}$. Given the limited number of GRBs with measured $A_{V, \mathrm{GRB}}$ we use an optical-to-X-ray spectral index of $\beta_{\mathrm{OX}}<0.4$ to identify those GRBs likely to have been significantly dustextinguished. We find that $50 \%$ of the hosts of dust-extinguished GRBs were detected, whereas the host of only one out of seven relatively unextinguished GRBs was detected with Herschel. Similarly, our results imply that the distribution in $A_{V, \mathrm{GRB}}$ is not the result of variations in the host galaxy inclination angle. If by-and-large the host galaxies of GRBs with $A_{V, \mathrm{GRB}}>1 \mathrm{mag}$ were viewed edge-on, and the hosts of relatively unextinguished GRBs were viewed face-on, then we would again expect the Herschel detection rate to be independent of $A_{V, \mathrm{GRB}}$.

Stellar mass is related to the galaxy dust mass, and is thus clearly an important parameter when considering the galaxy FIR emission. This is illustrated in Fig. 5, where we plot the stellar mass as a function of redshift for the combined sample of GRBHs. The apparent trend of increasing stellar mass with higher redshift is a result of selection effects, whereby high- $z$, low- $M_{*}$ galaxies are not generally detected at longer wavelengths. The dashed curve indicates the rough division within the $M_{*}-z$ parameter space between galaxies detected with Herschel (including GRBHs), and those that were undetected. Of those GRBHs below this line, 1/5 were detected, whereas above the line, $8 / 18$ were detected. When only considering the hosts of GRBs with $A_{V, \mathrm{GRB}}>1 \mathrm{mag}$, then only one lies below the curve,

5 The visual extinction along the line of sight to GRB 031203 is uncertain due to the large reddening within the Milky Way along the GRB line of sight. However, Prochaska et al. (2004) estimate $A_{V, \mathrm{GRB}} \sim 1$ mag. 


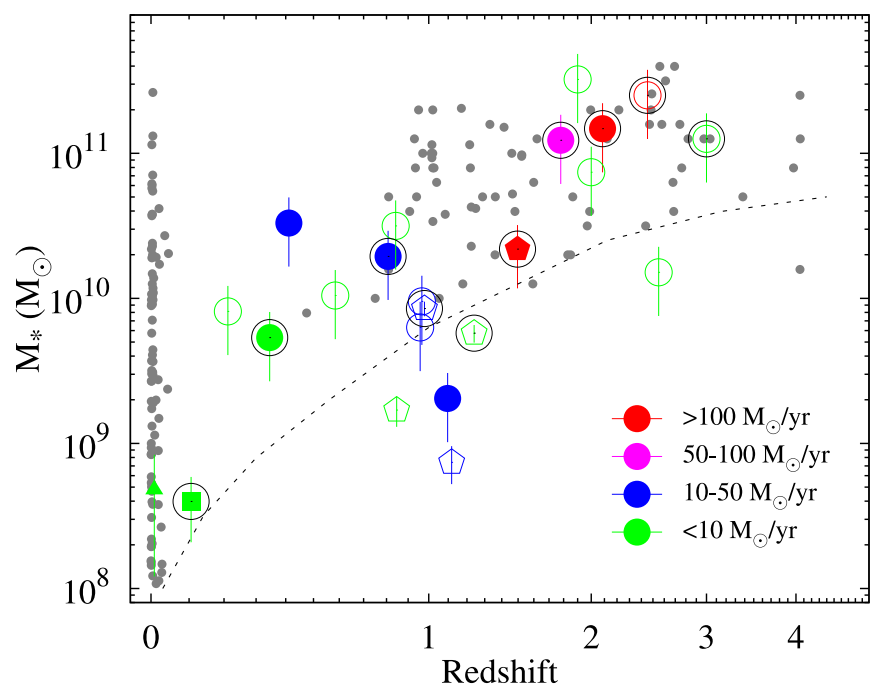

Fig. 5. Stellar mass against redshift for the sample of GRBHs from this work (pentagons), HPM14 (circles), and for GRBH 980425 (Michałowski et al. 2014, triangle) and GRBH 031203 (Symeonidis et al. 2014, square). The small grey circles correspond to a sample of non-GRBH targets observed and detected with Herschel (details in Sect. 5.2 and Figs. 6 and 7). Data points are colour-coded according to GRBH SFR. Those GRBHs detected with Herschel are plotted as filled symbols, and undetected GRBHs are shown with open symbols. GRBHs that had a GRB with $A_{V, \mathrm{GRB}}>1 \mathrm{mag}$ are indicated with an additional ring drawn around the data point. The dashed curve provides a rough divide between those galaxies detected and undetected with Herschel.

and this GRBH was undetected. Above the dashed curve, 6 out of 9 GRBHs of significantly extinguished GRBs were detected.

In this figure we also consider the SFR, which is known to correlate with the dust-to-stellar mass ratio (da Cunha et al. 2010; Calzetti 2001, and references therein). Detected GRBHs have progressively higher stellar mass and SFR as redshift increases, which is the combined result of the Malmquist bias and the downsizing of the star formation activity in progressively lower mass galaxies (i.e. the galaxy main sequence). For host galaxies with no FIR detections (open symbols), we use the dust corrected, optically derived SFR (measured from either emission lines or from optical/NIR SED fits), and thus these should be considered as lower limits.

Although using the afterglow line of sight dust extinction is, on the whole, a good diagnostic for identifying GRBs that reside within more massive and dust-rich hosts, there is clear scatter. This is to be expected, given that the light of the afterglow can travel through very different regions of the dusty media within the galaxy-disc plane and/or above it (e.g. Küpcü Yoldaş et al. 2007). Of those GRBs with $A_{V, \mathrm{GRB}}>1$ mag and with host galaxies that were not detected with Herschel, three (GRBHs 071021, 081109, 090926B) have stellar masses that are at the higher end of the GRB host galaxy distribution $\left(\log \left[M_{*} / M_{\odot}\right]>10\right)$, and they have relatively high SFRs $>10 M_{\odot} \mathrm{yr}^{-1}$. The non-detection of these galaxies may imply that the large afterglow extinction $\left(A_{V, \mathrm{GRB}}=1.5,3.4\right.$ and $1.4 \mathrm{mag}$, respectively $)$ arose predominantly from a dense and fairly isolated dust-cloud, or is associated with a line of sight that crosses the mid-plane of the disk (i.e. with an observed high inclination), rather than from a relatively dust-rich, galaxy-whole ISM. To place our sample in a broader context, in the next section we compare our results to the dust properties of other samples of galaxies observed with Herschel, covering a range of galaxy types and redshifts.

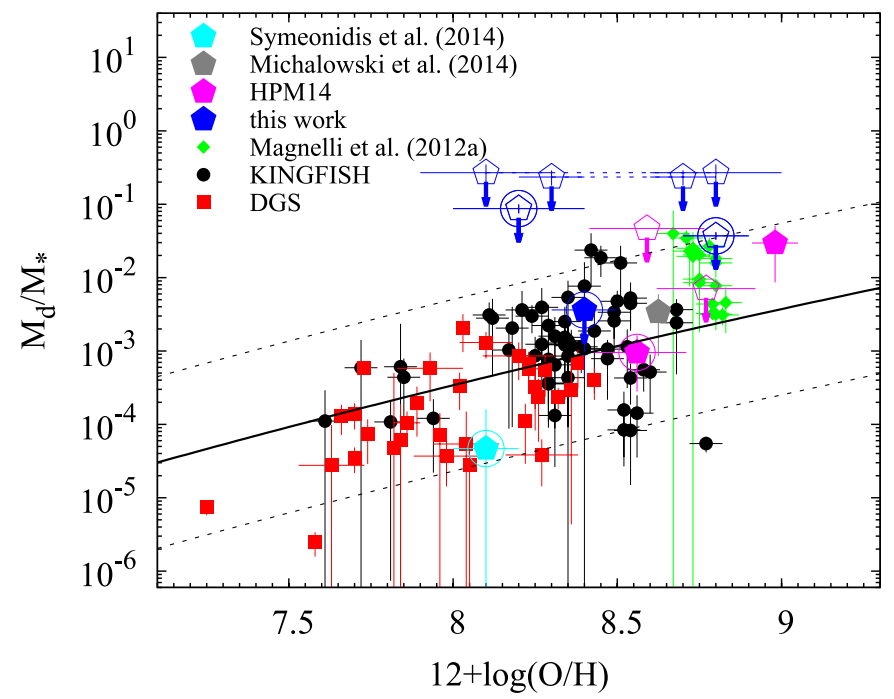

Fig. 6. $M_{\mathrm{d}} / M_{*}$ as a function of metallicity for a sample of nearby galaxies taken from the KINGFISH (black filled circles; Kennicutt et al. 2011), and the DGS (red filled squares; Madden et al. 2013) Herschel guaranteed time key projects, and a sample of high redshift $(z>1)$, star forming galaxies from Magnelli et al. (2012a) (green filled diamonds). Metallicities of the $z>1$ star forming galaxies were estimated using the mass-metallicity relation and converted into the Denicoló et al. (2002) system. GRBH 031203 (Symeonidis et al. 2014, cyan pentagon), GRBH 980425 (Michałowski et al. 2014, grey pentagon), and the subset of GRBHs with known metallicity from HPM14 (pink pentagons; GRBHs 980703, 020819B, 050223, 051022) and from this paper (blue pentagons) are also plotted. Filled symbols correspond to Herschel detections, and upper limits are shown as downward arrows. A ring around the data point indicates galaxies that hosted a GRB with afterglow extinction $A_{V, G R B}>1 \mathrm{mag}$. The solid black line is the best-fit power law to the combined KINGFISH and DGS data taken from Rémy-Ruyer et al. (2013), and the dashed lines represent the $3 \sigma$ dispersion.

\subsection{Dust to stellar mass ratio}

Rémy-Ruyer et al. (2013) combined the sample of nearby galaxies from DGS and KINGFISH and found a positive correlation between the dust-to-stellar mass ratio and galaxy metallicity. In both samples the metallicity was derived using the $R_{23}$ ratio and applying the empirical calibration of Pilyugin \& Thuan (2005). In Fig. 6 we reproduce this plot and include a sample of star forming galaxies at $z>1$, and the sample of $\mathrm{GRBHs}^{6}$. A range of methods have been used to estimate the metallicities for the GRBHs, depending on the available data. The metallicities for GRBH 000210 and GRBH 000418 (Piranomonte et al., in prep.) and GRBH 070306 (Vergani et al., in prep.) were all derived using the $R_{23}$ ratio and applying the calibration from Kobulnicky \& Kewley (2004). In the cases where we were unable to select between the lower and higher branch solutions, both metallicities are shown in Fig. 6, and are connected with a dotted line. The metallicities for GRBH 081109 and 090926B (Krühler et al., in prep.) were based on a number of line ratio diagnostics from Nagao et al. (2006), as were the metallicities for those GRBHs in HPM14 (Mannucci et al. 2011).

All GRBHs detected with Herschel (from this work and from HPM14) have $M_{\mathrm{d}} / M_{*}$ ratios that are within the $3 \sigma$ dispersion of the DGS and KINGFISH samples (dashed lines), and those GRBHs with only upper limits on their dust mass have

6 In the case of GRBH 070306, which is present in both this work and in HPM14, we use the values reported in this paper. 


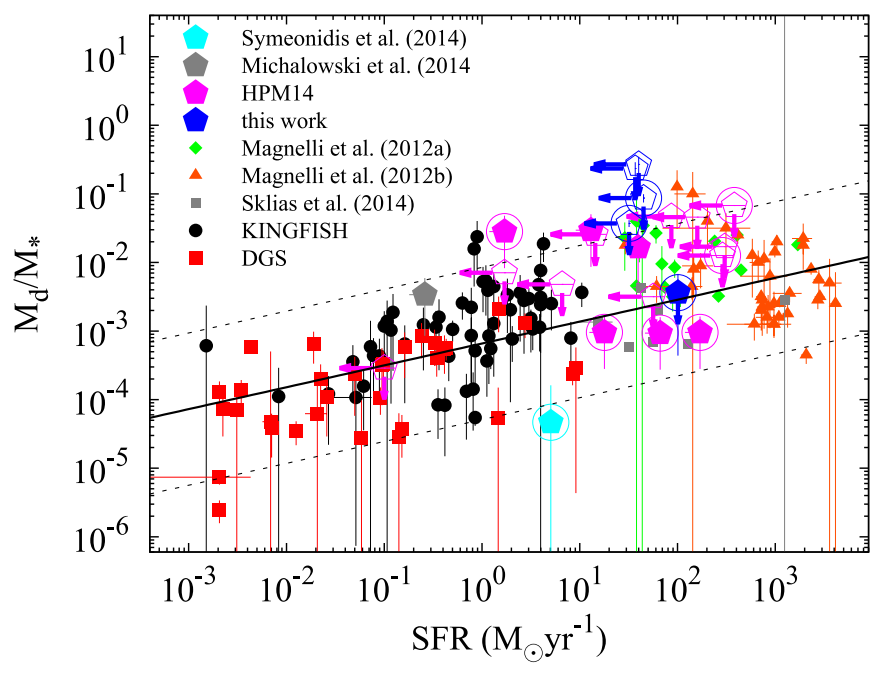

Fig. 7. $M_{\mathrm{d}} / M_{*}$ as a function of SFR. The same galaxy samples are shown as in Fig. 6, in addition to a sample of star forming lensed galaxies from Sklias et al. (2014; small grey squares), and a sample of SMGs from Magnelli et al. (2012b; orange triangles). The solid black line is our best-fit power law to all galaxy samples combined, not including the GRBH sample $\left(M_{\mathrm{d}} / M_{*}=0.001 \times S F R^{0.3}\right)$, and the dashed lines correspond the $3 \sigma$ dispersion.

dust-to-stellar mass ratios that are consistent with the predicted value for their given metallicity. The scatter about the line-ofbest-fit shown in Fig. 6 is large, and with our current Herschel limits we cannot rule out that the undetected GRBHs have unusually low dust-to-stellar mass ratios. However, the general consistency between the GRBH data points and the best-fit relation between $M_{\mathrm{d}} / M_{*}$ and $12+\log (\mathrm{O} / \mathrm{H})$ implies that the low detection rate of GRBHs is a result of the generally low stellar mass of the sample relative to that of other submm-detected galaxies at similar redshifts.

In Fig. 7 we plot the dust-to-stellar mass ratio as a function of SFR, where the SFR is derived using FIR data, and for the nondetected GRBHs is therefore an effective upper limit. This plot shows the general trend of increasing dust-to-stellar mass ratio with SFR across all galaxy types (da Cunha et al. 2010; Calzetti 2001, and references therein). As before, we find that all our non-detected GRBHs have upper limits that lie either above or on the general relation followed by the other galaxy populations (solid line). Those GRBHs that were detected all lie within the region of space occupied by the high- $z$ galaxy samples, all of which have $S F R \gtrsim 10 M_{\odot} \mathrm{yr}^{-1}$. However, they have lower SFRs than the majority of the SMG sample.

Given the dependence between dust temperature and mass, we re-fit all GRBHs assuming the same dust-temperature measured for GRBH $070306(T=50 \mathrm{~K})$ to see how this affected our dust mass upper limits. We found that the dust mass limits decreased in some cases by a factor of 8 or 9 . This nevertheless still not does bring the dust-to-mass ratio of our undetected GRBHs below the $3 \sigma$ dispersions shown in Figs. 6 and 7, and thus our conclusions are broadly unchanged.

\section{Summary}

We selected a small sample of five GRBHs with evidence of having a relatively large dust mass, and used Herschel PACS and SPIRE observations to sample the peak of the dust emission within these galaxies. Despite the sensitivity of Herschel, we only detected one GRBH, which had the largest amount of visual extinction along the GRB line of sight and a relatively high stellar mass. In order to improve our statistics, we combined our sample with the GRBHs from HPM14, Michałowski et al. (2014), and Symeonidis et al. (2014), all also observed with Herschel. We found a sizeable increase in the Herschel detection rate when we only considered those galaxies that hosted GRBs with considerably dust-extinguished afterglows. This implies that the bulk of the afterglow extinguishing dust resides within the ISM of the host galaxy rather than within discrete, dense clouds.

In addition we found that the dust-to-stellar mass ratios and limits of GRBHs are consistent with other star forming galaxy populations selected by different means. Our results thus indicate that the FIR non-detection rate of $60-80 \%$ within GRBHs is due to the combination of relatively high redshift and low stellar mass of our galaxies. It is possible that metallicity also plays a role, with lower metallicity galaxies of a given stellar mass having lower dust masses than their higher metallicity counterparts (see Fig. 6). However, a larger number of GRBH metallicity measurements would be needed to investigate this further.

Herschel observations of GRBHs have provided the first irrefutable detections of GRBHs at submm wavelengths, and the most accurate sampling of the thermal dust emission peak of these galaxies to date. The full SED coverage provided by optical through to FIR and submm wavelengths with Herschel and on-going observatories such as ALMA and JWST enable the properties of GRBHs to be fully characterised, thus resulting in a more complete understanding of the range in environmental properties present within GRBHs.

Acknowledgements. We thank A. Rémy-Ruyer for sharing their best-fit $M_{\mathrm{d}}$ values to the DFG sample, and Francesco Ritacca and the anonymous referee for constructive comments. P.S. acknowledges support through the Sofja Kovalevskaja Award from the Alexander von Huboldt Foundation of Germany. S.S. acknowledges support from the Bundesministerium für Wirtschaft and Technologie through DLR (Deutsches Zentrum für Luft- ind Raumfahrt e.V.). L.K.H. and E.P. are grateful to support from PRIN-INAF 2012/13. D.P. acknowledges the kind hospitality at the MPE and financial support from the OCEVU LabEx. Part of the funding for GROND (both hardware as well as personel) was granted from the Leibniz-Prize to Prof. G. Hasinger (DFG grant HA 1850/28-1). This study is based on data acquired with the Atacama Pathfinder Experiment (APEX), programme ID M-087.F-0024-2011, and the Herschel observatory, programme ID OT2_ppschady_2. APEX is a collaboration between the Max-Planck-Insitut für Radioastronomie, the European Southern Observatory, and the Onsala Space Observatory. The Herschel spacecraft was designed, built, tested, and launched under a contract to ESA managed by the Herschel/Planck Project team by an industrial consortium under the overall responsibility of the prime contractor Thales Alenia Space (Cannes), and including Astrium (Friedrichshafen) responsible for the payload module and for system testing at spacecraft level, Thales Alenia Space (Turin) responsible for the service module, and Astrium (Toulouse) responsible for the telescope, with in excess of a hundred subcontractors. PACS has been developed by a consortium of institutes led by MPE (Germany) and including UVIE (Austria); KU Leuven, CSL, IMEC (Belgium); CEA, LAM (France); MPIA (Germany); INAF-IFSI/OAA/OAP/OAT, LENS, SISSA (Italy); IAC (Spain). This development has been supported by the funding agencies BMVIT (Austria), ESA-PRODEX (Belgium), CEA/CNES (France), DLR (Germany), ASI/INAF (Italy), and CICYT/MCYT (Spain). SPIRE has been developed by a consortium of institutes led by Cardiff University (UK) and including Univ. Lethbridge (Canada); NAOC (China); CEA, LAM (France); IFSI, Univ. Padua (Italy); IAC (Spain); Stockholm Observatory (Sweden); Imperial College London, RAL, UCL-MSSL, UKATC, Univ. Sussex (UK); and Caltech, JPL, NHSC, Univ. Colorado (USA). This development has been supported by national funding agencies: CSA (Canada); NAOC (China); CEA, CNES, CNRS (France); ASI (Italy); MCINN (Spain); SNSB (Sweden); STFC (UK); and NASA (USA). HIPE is a joint development by the Herschel Science Ground Segment Consortium, consisting of ESA, the NASA Herschel Science Center, and the HIFI, PACS and SPIRE consortia. 


\section{Appendix A}

Table A.1. Optical ( $U$ to $Z$ ) photometric measurements of pre-Swift GRB host galaxies.

\begin{tabular}{lcccccc}
\hline \hline GRB Host & $U$ & $B$ & $V$ & $R$ & $I$ & $Z$ \\
\hline $000210^{1}$ & $24.13_{-0.13}^{+0.13}$ & $24.21_{-0.13}^{+0.13}$ & $24.17_{-0.08}^{+0.08}$ & $23.62_{-0.10}^{+0.10}$ & $22.89_{-0.12}^{+0.12}$ & $23.35_{-0.28}^{+0.28}$ \\
$000418^{2}$ & $24.36_{-0.30}^{+0.30}$ & $23.94_{-0.05}^{+0.05}$ & $23.80_{-0.06}^{+0.06}$ & $23.65_{-0.05}^{+0.05}$ & $23.25_{-0.05}^{+0.05}$ & $23.01_{-0.10}^{+0.10}$ \\
\hline
\end{tabular}

Notes. All magnitudes are AB and have been corrected for Galactic reddening (Schlafly \& Finkbeiner 2011). All magnitudes in the AB system and are corrected for Galactic foreground reddening.

References. ${ }^{(1)}$ Gorosabel et al. (2003a); (2) Gorosabel et al. (2003b) .

Table A.2. Optical $\left(u^{\prime}\right.$ to $\left.z^{\prime}\right)$ photometric measurements of Swift GRB host galaxies.

\begin{tabular}{lccccccccc}
\hline \hline GRB Host & $u^{\prime}$ & $U$ & $g^{\prime}$ & $V$ & $r^{\prime}$ & $R$ & $i^{\prime}$ & $I$ & $z^{\prime}$ \\
\hline $070306^{1}$ & $23.05_{-0.46}^{+0.46}$ & - & $22.81_{-0.09}^{+0.09}$ & - & $23.02_{-0.09}^{+0.09}$ & $22.94_{-0.09}^{+0.09}$ & $22.76_{-0.13}^{+0.13}$ & $22.58_{-0.19}^{+0.19}$ & $22.83_{-0.17}^{+0.17}$ \\
$081109^{2}$ & - & $23.15_{-0.14}^{+0.14}$ & $23.01_{-0.07}^{+0.07}$ & $22.80_{-0.06}^{+0.06}$ & $22.70_{-0.07}^{+0.07}$ & - & $21.98_{-0.08}^{+0.08}$ & $21.93_{-0.09}^{+0.09}$ & $21.97_{-0.09}^{+0.09}$ \\
$090926 \mathrm{~B}^{2}$ & - & $23.61_{-0.13}^{+0.13}$ & $23.23_{-0.07}^{+0.07}$ & - & $22.90_{-0.06}^{+0.06}$ & - & $22.88_{-0.12}^{+0.12}$ & - & $22.41_{-0.10}^{+0.10}$ \\
\hline
\end{tabular}

Notes. All values are as in Table A.1.

References. ${ }^{(1)} u^{\prime}$ and $I$ magnitudes from Jaunsen et al. (2008). All other magnitudes from Krühler et al. (2011); ${ }^{(2)}$ Krühler et al. (2011) .

Table A.3. NIR ( $Y$ to $K$ ) photometric measurements of GRB host galaxies.

\begin{tabular}{lccccccc}
\hline \hline GRB Host & $Y$ & HST/F110 & $J$ & HST/F125 & HST/F160 & $H$ & $K$ \\
\hline $000210^{1}$ & - & - & $22.90_{-0.10}^{+0.10}$ & - & - & $22.90_{-0.23}^{+0.23}$ & $22.80_{-0.14}^{+0.14}$ \\
$000418^{2}$ & - & - & $23.21_{-0.10}^{+0.10}$ & - & - & - & $23.06_{-0.30}^{+0.30}$ \\
$070306^{3}$ & & - & $21.60_{-0.08}^{+0.08}$ & $21.89_{-0.03}^{+0.03}$ & $21.68_{-0.03}^{+0.03}$ & $21.19_{-0.12}^{+0.12}$ & $21.37_{-0.10}^{+0.10}$ \\
$081109^{3}$ & $21.61_{-0.08}^{+0.08}$ & $21.50_{-0.03}^{+0.03}$ & $21.36_{-0.06}^{+0.06}$ & - & $21.28_{-0.03}^{+0.03}$ & $21.49_{-0.4}^{+0.4}$ & $21.04_{-0.08}^{+0.08}$ \\
$090926 B^{3}$ & - & - & $21.86_{-0.13}^{+0.13}$ & - & - & $21.9_{-0.3}^{+0.3}$ & $21.43_{-0.19}^{+0.19}$ \\
\hline
\end{tabular}

Notes. All values are as in Table A.1.

References. ${ }^{(1)}$ Gorosabel et al. (2003a); ${ }^{(2)}$ Gorosabel et al. (2003b); ${ }^{(3)}$ HST magnitudes from Perley et al. (2013). All other magnitudes from Krühler et al. (2011).

Table A.4. Spitzer photometric measurements of GRB host galaxies.

\begin{tabular}{l|cccc}
\hline \hline & \multicolumn{4}{|c}{ Flux density $(\mu \mathrm{Jy})$} \\
GRB Host & $3.6 \mu \mathrm{m}$ & $4.5 \mu \mathrm{m}$ & $8.0 \mu \mathrm{m}$ & $24.0 \mu \mathrm{m}$ \\
\hline $000210^{1}$ & - & $<6.3$ & $15.0 \pm 5.1$ & $<31.5$ \\
$000418^{2}$ & - & $4.8_{-1.8}^{+1.8}$ & - & - \\
$070306^{3}$ & $10.65_{-0.48}^{+0.48}$ & $12.28_{-0.59}^{+0.59}$ & - & - \\
$081109^{3}$ & $18.88_{-1.26}^{+1.26}$ & $15.70_{-1.36}^{+1.36}$ & - & - \\
$090926 \mathrm{~B}^{4}$ & $10.5_{-0.5}^{+0.5}$ & $7.4_{-0.4}^{+0.4}$ & - & - \\
\hline
\end{tabular}

Notes. Upper limits are given at $3 \sigma$ confidence.

References. ${ }^{(1)}$ Michałowski et al. (2008); ${ }^{(2)}$ Castro Cerón et al. (2010); (3) Perley et al. (2013); ${ }^{(4)}$ this work.

Table A.5. Submillimetre photometric measurements of GRB host galaxies.

\begin{tabular}{lcc}
\hline \hline & \multicolumn{2}{c}{ Flux density (mJy) } \\
GRB Host & $450 \mu \mathrm{m}$ & $850 \mu \mathrm{m}$ \\
\hline $000210^{1}$ & $<92.4$ & $2.97_{-0.88}^{+0.88}$ \\
$000418^{1}$ & $<56.76$ & $3.15_{-0.90}^{+0.90}$ \\
070306 & - & $<7.44$ \\
081109 & $<13.2$ & $<14.1$ \\
$090926 \mathrm{~B}$ & - & $<14.64$ \\
\hline
\end{tabular}

Notes. Upper limits are given at $3 \sigma$ confidence.

References. ${ }^{(1)}$ Berger et al. (2003). 


\section{References}

Arnouts, S., Cristiani, S., Moscardini, L., et al. 1999, MNRAS, 310, 540

Baldry, I. K., \& Glazebrook, K. 2003, ApJ, 593, 258

Balog, Z., Müller, T., Nielbock, M., et al. 2013, Exp. Astron., 38

Berger, E., Diercks, A., Frail, D. A., et al. 2001, ApJ, 556, 556

Berger, E., Cowie, L. L., Kulkarni, S. R., et al. 2003, ApJ, 588, 99

Bianchi, S. 2013, A\&A, 552, A89

Bloom, J. S., Berger, E., Kulkarni, S. R., Djorgovski, S. G., \& Frail, D. A. 2003, AJ, 125, 999

Bloom, J. S., Starr, D. L., Blake, C. H., Skrutskie, M. F., \& Falco, E. E. 2006, Astronomical Data Analysis Software and Systems XV, 351, 751

Bruzual, G., \& Charlot, S. 2003, MNRAS, 344, 1000

Burrows, D. N., Hill, J. E., Nousek, J. A., et al. 2005, Space Sci. Rev., 120, 165

Butler, N. R., Bloom, J. S., \& Poznanski, D. 2010, ApJ, 711, 495

Butler, N., Klein, C., Fox, O., et al. 2012, Proc. SPIE, 8446

Calzetti, D. 2001, PASP, 113, 1449

Calzetti, D., Armus, L., Bohlin, R. C., et al. 2000, ApJ, 533, 682

Casey, C. M. 2012, MNRAS, 425, 3094

Castro Cerón, J. M., Michałowski, M. J., Hjorth, J., et al. 2010, ApJ, 721, 1919

Chabrier, G. 2003, PASP, 115, 763

da Cunha, E., Eminian, C., Charlot, S., \& Blaizot, J. 2010, MNRAS, 403, 1894

Dale, D. A., Aniano, G., Engelbracht, C. W., et al. 2012, ApJ, 745, 95

D'Avanzo, P., Covino, S., Antonelli, L. A., et al. 2008, GRB Coordinates Network, 8501, 1

Denicoló, G., Terlevich, R., \& Terlevich, E. 2002, MNRAS, 330, 69

Dunne, L., \& Eales, S. A. 2001, MNRAS, 327, 697

Elliott, J., Krühler, T., Greiner, J., et al. 2013, A\&A, 556, A23

Fruchter, A. S., Levan, A. J., Strolger, L., et al. 2006, Nature, 441, 463

Fynbo, J. P. U., Malesani, D., Jakobsson, P., \& D’Elia, V. 2009, GRB Coordinates

Network, 9947, 1

Galama, T. J., Vreeswijk, P. M., van Paradijs, J., et al. 1999, A\&A, 138, 465

Gehrels, N., Chincarini, G., Giommi, P., et al. 2004, ApJ, 611, 1005

Gorosabel, J., Christensen, L., Hjorth, J., et al. 2003a, A\&A, 400, 127

Gorosabel, J., Klose, S., Christensen, L., et al. 2003b, A\&A, 409, 123

Graham, J. F., \& Fruchter, A. S. 2013, ApJ, 774, 119

Graham, J. F., Fruchter, A. S., Kewley, L. J., et al. 2009, AIP Conf. Ser., 1133, 269

Greiner, J., Bornemann, W., Clemens, C., et al. 2008, PASP, 120, 405

Greiner, J., Krühler, T., Klose, S., et al. 2011, A\&A, 526, A30

Griffin, M. J., Abergel, A., Abreu, A., et al. 2010, A\&A, 518, L3

Güsten, R., Nyman, L. A., Schilke, P., et al. 2006, A\&A, 454, L13

Hjorth, J., Sollerman, J., Møller, P., et al. 2003, Nature, 423, 847

Holland, W. S., Robson, E. I., Gear, W. K., et al. 1999, MNRAS, 303, 659

Hunt, L., Palazzi, E., Rossi, A., et al. 2011, ApJ, 736, L36

Hunt, L., Palazzi, E., Michałowski, M. J., et al. 2014, A\&A, 565, A112 (HPM14)

Ilbert, O., Arnouts, S., McCracken, H. J., et al. 2006, A\&A, 457, 841

Ilbert, O., Salvato, M., Le Floc'h, E., et al. 2010, ApJ, 709, 644

Jakobsson, P., Hjorth, J., Fynbo, J. P. U., et al. 2004, ApJ, 617, L21

Jaunsen, A. O., Rol, E., Watson, D. J., et al. 2008, ApJ, 681, 453

Kajisawa, M., Ichikawa, T., Tanaka, I., et al. 2009, ApJ, 702, 1393

Kelly, P. L., Filippenko, A. V., Modjaz, M., \& Kocevski, D. 2014, ApJ, 789, 23

Kennicutt, R. C., Jr. 1998, ARA\&A, 36, 189

Kennicutt, R. C., Calzetti, D., Aniano, G., et al. 2011, PASP, 123, 1347

Kewley, L. J., \& Ellison, S. L. 2008, ApJ, 681, 1183

Kistler, M. D., Yüksel, H., Beacom, J. F., Hopkins, A. M., \& Wyithe, J. S. B. 2009, ApJ, 705, L104

Klose, S., Stecklum, B., Masetti, N., et al. 2000, ApJ, 545, 271

Kobulnicky, H. A., \& Kewley, L. J. 2004, ApJ, 617, 240
Kocevski, D., \& West, A. A. 2011, ApJ, 735, L8

Krühler, T., Greiner, J., Schady, P., et al. 2011, A\&A, 534, A108

Küpcü Yoldaş, A., Salvato, M., Greiner, J., et al. 2007, A\&A, 463, 893

Langer, N., \& Norman, C. A. 2006, ApJ, 638, L63

Levesque, E. M., Kewley, L. J., Berger, E., \& Zahid, H. J. 2010a, AJ, 140, 1557

Levesque, E. M., Kewley, L. J., Graham, J. F., \& Fruchter, A. S. 2010b, ApJ, 712, L26

Lo Faro, B., Franceschini, A., Vaccari, M., et al. 2013, ApJ, 762, 108

MacFadyen, A. I., \& Woosley, S. E. 1999, ApJ, 524, 262

Madden, S. C., Rémy-Ruyer, A., Galametz, M., et al. 2013, PASP, 125, 600

Magdis, G. E., Daddi, E., Béthermin, M., et al. 2012, ApJ, 760, 6

Magdis, G. E., Rigopoulou, D., Helou, G., et al. 2013, A\&A, 558, A136

Magnelli, B., Saintonge, A., Lutz, D., et al. 2012a, A\&A, 548, A22

Magnelli, B., Lutz, D., Santini, P., et al. 2012b, A\&A, 539, A155

Malesani, D., Fynbo, J. P. U., \& D’Elia, V. 2009, GRB Coordinates Network, 9944, 1

Mannucci, F., Salvaterra, R., \& Campisi, M. A. 2011, MNRAS, 414, 1263

Michałowski, M. J., Hjorth, J., Castro Cerón, J. M., \& Watson, D. 2008, ApJ, 672,817

Michałowski, M., Hjorth, J., \& Watson, D. 2010, A\&A, 514, A67

Michałowski, M. J., Dunlop, J. S., Cirasuolo, M., et al. 2012a, A\&A, 541, A85

Michałowski, M. J., Kamble, A., Hjorth, J., et al. 2012b, ApJ, 755, 85

Michałowski, M. J., Hunt, L. K., Palazzi, E., et al. 2014, A\&A, 562, A70

Mitchell, P. D., Lacey, C. G., Baugh, C. M., \& Cole, S. 2013, MNRAS, 435, 87

Modjaz, M., Kewley, L., Kirshner, R. P., et al. 2008, AJ, 135, 1136

Nagao, T., Maiolino, R., \& Marconi, A. 2006, A\&A, 459, 85

Ott, S. 2010, Astronomical Data Analysis Software and Systems XIX, 434, 139

Pearson, C., Lim, T., North, C., et al. 2013, Exp. Astron., 37

Peng, C. Y., Ho, L. C., Impey, C. D., \& Rix, H.-W. 2002, AJ, 124, 266

Pérez-González, P. G., Egami, E., Rex, M., et al. 2010, A\&A, 518, L15

Perley, D. A., Levan, A. J., Tanvir, N. R., et al. 2013, ApJ, 778, 128 (PLT13)

Pilbratt, G. L., Riedinger, J. R., Passvogel, T., et al. 2010, A\&A, 518, L1

Pilyugin, L. S., \& Thuan, T. X. 2005, ApJ, 631, 231

Piro, L., Frail, D. A., Gorosabel, J., et al. 2002, ApJ, 577, 680

Poglitsch, A., Waelkens, C., Geis, N., et al. 2010, A\&A, 518, L2

Pozzetti, L., Bolzonella, M., Lamareille, F., et al. 2007, A\&A, 474, 443

Prochaska, J. X., Bloom, J. S., Chen, H.-W., et al. 2004, ApJ, 611, 200

Rémy-Ruyer, A., Madden, S. C., Galliano, F., et al. 2013, A\&A, 557, A95

Robertson, B. E., \& Ellis, R. S. 2012, ApJ, 744, 95

Rol, E., Levan, A., Tanvir, N., Schirmer, M., \& Castro-Tirado, A. J. 2007, GRB Coordinates Network, 6174, 1

Rossi, A., Klose, S., Ferrero, P., et al. 2012, A\&A, 545, A77

Salpeter, E. E. 1955, ApJ, 121, 161

Savaglio, S., Glazebrook, K., \& Le Borgne, D. 2009, ApJ, 691, 182 (SGL09)

Savaglio, S., Rau, A., Greiner, J., et al. 2012, MNRAS, 420, 627

Schady, P., Mason, K. O., Page, M. J., et al. 2007, MNRAS, 377, 273

Schady, P., Dwelly, T., Page, M. J., et al. 2012, A\&A, 537, A15

Schlafly, E. F., \& Finkbeiner, D. P. 2011, ApJ, 737, 103

Silva, L., Granato, G. L., Bressan, A., \& Danese, L. 1998, ApJ, 509, 103

Siringo, G., Kreysa, E., Kovács, A., et al. 2009, A\&A, 497, 945

Skibba, R. A., Engelbracht, C. W., Dale, D., et al. 2011, ApJ, 738, 89

Sklias, P., Zamojski, M., Schaerer, D., et al. 2014, A\&A, 561, A149

Svensson, K. M., Levan, A. J., Tanvir, N. R., et al. 2012, MNRAS, 421, 25

Symeonidis, M., Oates, S. R., de Pasquale, M., et al. 2014, MNRAS, 443, 124

Tanvir, N. R., Barnard, V. E., Blain, A. W., et al. 2004, MNRAS, 352, 1073

Wanderman, D., \& Piran, T. 2010, MNRAS, 406, 1944

Wolf, C., \& Podsiadlowski, P. 2007, MNRAS, 375, 1049

Wuyts, S., Franx, M., Cox, T. J., et al. 2009, ApJ, 696, 348 